Original Paper http://ajol.info/index.php/ijbcs $\quad$ http://indexmedicus.afro.who.int

\title{
Révision du genre Digitaria Haller (Poaceae) au Sénégal : proposition d'une clé de détermination pour une meilleure identification des espèces
}

\author{
Ablaye NGOM $^{1 *}$, Mame Samba MBAYE ${ }^{1}$, Adeline BARNAUD ${ }^{2,3}$, Aboubacry KANE ${ }^{1}$, \\ Ngasoumana BA ${ }^{1}$, Madiop GUEYE ${ }^{1}$, Abdoul Aziz CAMARA ${ }^{1}$, Yacine Badiane NDOUR ${ }^{3}$, \\ et Kandioura NOBA ${ }^{1}$ \\ ${ }^{I}$ Département de Biologie Végétale, Faculté des Sciences et Techniques, Université Cheikh Anta DIOP, B.P. \\ 5005 Dakar-Fann, Sénégal. \\ ${ }^{2}$ Institut de Recherche pour le Développement-UMR DIADE, Montpellier, France. \\ ${ }^{3}$ Laboratoire National de Recherches sur les Productions Végétales/Institut Sénégalais de Recherches \\ Agricoles, Sénégal. \\ *Auteur correspondant, E-mail : ngomito@hotmail.com ; Tel : (221) $775550027 /(221) 338223259$
}

\section{RESUME}

Le genre Digitaria Haller (Poaceae) constitue l'un des genres les plus diversifiés des Poaceae du Sénégal avec 19 espèces. Bien qu'il y ait une monographie mondiale du genre, la détermination des espèces reste difficile et repose essentiellement sur les caractères de l'appareil reproducteur. Ce travail, qui est une révision du genre Digitaria au Sénégal, est entrepris afin de rechercher des caractères pertinents sur l'appareil végétatif et d'améliorer l'identification des espèces. Pour ce faire, une description détaillée des différents organes des spécimens étudiés est réalisée à l'aide d'une loupe binoculaire. Les résultats de l'étude montrent que la pubescence de l'épillet est un caractère essentiel pour l'identification des espèces. De plus, les longueurs relatives de la glume supérieure et de la lemma inférieure sont très intéressantes pour la discrimination d'espèces affines. Toutefois, l'étude supplémentaire des organes végétatifs de la plante adulte montre que certains caractères végétatifs pourraient contribuer considérablement à l'amélioration de l'identification des espèces. Il s'agit du cycle biologique, de la pubescence de la ligule, de la marge du limbe, des nœuds et des entre-nœuds du chaume qui sont des caractères plus faciles à observer. Une clé de détermination basée sur des caractères végétatifs et reproducteurs des espèces est ainsi élaborée.

(C) 2015 International Formulae Group. All rights reserved.

Mots clés : Digitaria, Poaceae, révision, clé de détermination, Sénégal.

\section{Review of Digitaria Haller (Poaceae) genus in Senegal: suggestion of a determination key for a better identification of species}

\begin{abstract}
The genus Digitaria Haller (Poaceae) is one of the most diversified genera of Poaceae in Senegal with 19 species. Although it has a worldwide monograph of the genus, the identification of species remains difficult and mainly based on the characteristics of the reproductive system. This work, which is a review of the genus
\end{abstract}


Digitaria in Senegal, is undertaken in order to search relevant characteristics on the vegetative system and to improve the identification of species. To do this, a detailed description of the various organs of the studied specimens is carried out using a binocular microscope. The results of the study show that the pubescence of the spikelet is an essential characteristic for the identification of species. Moreover, the relative lengths of the upper glume and lower lemma are very interesting for the discrimination of species closely related. However, the additional study of vegetative organs of the adult plant shows that some vegetative characteristics could contribute considerably to the improvement of the identification of species. It is the biological cycle, the pubescence of the ligule, the margin of the leaf blade, nodes and internodes of the thatch which are characteristics easier to observe. A key of determination based on vegetative and reproductive characteristics of species is thus worked out.

(C) 2015 International Formulae Group. All rights reserved.

Mots clés: Digitaria, Poaceae, review, key of determination, Senegal.

\section{INTRODUCTION}

Le genre Digitaria Haller compte environ 230 espèces réparties principalement dans les régions tropicales et subtropicales, rarement dans les régions tempérées des deux hémisphères (Vega et Rúgolo de Agrasar, $2002 \mathrm{~b}$; Adoukonou-Sagbadja et al., 2006 ; Merchán, 2006, Vega et al., 2009). Le genre Digitaria est un genre d'une grande importance sur le plan économique. Il renferme en effet des espèces fourragères et des espèces adventices (Noba, 2002 ; Quattrocchi, 2006; Bassène et al., 2012, Mbaye, 2013 ; Bassène et al., 2014). Au plan scientifique, certaines espèces comme $D$. aristulata et $D$. gentilis sont signalées comme des espèces endémiques du Sénégal (Bâ et Noba, 2001 ; USAID/Sénégal, 2008).

Le genre Digitaria, du latin digitus ou doigt, allusion à l'inflorescence digitée (Hooker, 2009), est reconnu comme l'un des genres les plus complexes de la tribu des Paniceae. Cette difficulté taxonomique est liée au grand nombre d'espèces, leur large répartition géographique, la complexité des caractères et à la mauvaise connaissance des relations interspécifiques. Le genre est caractérisé par ses épillets regroupés par 2, 3 ou 4 , sa glume inférieure réduite ou absente ainsi que la paléole de sa fleur supérieure qui serre complètement la lemma supérieure (Hooker, 2009) cartilagineuse et glabre et dont les marges sont membraneuses, généralement hyalines et repliées sur la paléa supérieure (Giraldo-Cañas, 2004 ; Merchán, 2006).
Au Sénégal, 19 espèces ont été répertoriées suite à la synthèse des travaux de Berhaut (1967) et de Vanden Berghen (1991) couplée aux données recueillies à l'herbier de l'IFAN (Institut Fondamental d'Afrique Noire). La clé de détermination qui existe (Berhaut, 1967) ne concerne que 12 de ces espèces. De plus, leur identification repose essentiellement sur la morphologie de l'épillet qui est caractéristique (Hooker, 2009) mais n'apparaît qu'au stade reproducteur. Il est donc important de rechercher d'autres caractères discriminants pertinents susceptibles de faciliter l'identification des espèces à tous les stades de développement. C'est dans cette optique que ce présent travail est entrepris pour: 1- déterminer les caractères végétatifs et reproducteurs les plus discriminants et les plus spécifiques et 2proposer une clé de détermination de toutes les espèces présentes au Sénégal.

\section{MATERIEL ET METHODES}

Le matériel végétal provient des Herbiers de DAKAR (Département de Biologie Végétale) et de l'IFAN (Institut Fondamental d'Afrique Noire). Une description détaillée de la plante adulte est réalisée et porte sur des échantillons conservés dans ces herbiers et sur des échantillons fraîchement collectés. Des informations issues des travaux de Poilecot $(1995,1999)$ et Vanden Berghen (1991) ont permis de compléter la description de chacune des espèces. 
Sur les individus étudiés, des observations à l'œil nu ou à la loupe binoculaire ont été effectuées et portaient sur :

- les caractères morphologiques qualitatifs de certains organes tels que :

- le chaume (cycle biologique, port, pubescence),

- la feuille : gaine (pubescence), ligule (aspect, forme, pubescence) et le limbe (forme, pubescence, etc.),

- l'inflorescence générale (type, organisation des racèmes, forme du rachis, marge du rachis),

- les épillets (forme, pubescence),

- les glumes (forme, pubescence),

- les fleurs (forme, pubescence),

- $\quad$ et le fruit (type, forme, couleur).

- les caractères morphologiques quantitatifs (groupement, hauteur, longueur, largeur, nombre, etc.) des différents organes précités.

Les résultats de ces observations sont consignés dans un tableau brut qui est, par la suite, scindé en plusieurs tableaux simplifiés classés par organe. La nomenclature utilisée dans ce travail est basée sur les travaux de Lebrun et Stork (1991).

\section{RESULTATS}

\section{Le chaume}

L'étude morphologique du chaume a porté sur les caractères tels que le cycle biologique, la hauteur, le port (allure et ramification) et sur la pubescence des nœuds et des entre-nœuds.

L'analyse du Tableau 1 montre que les chaumes de Digitaria présentent des caractères différentiels qui permettent une meilleure identification de ces espèces.

En effet, en ce qui concerne le cycle biologique, le genre Digitaria est représenté au Sénégal par des herbes annuelles à l'exception de $D$. diagonalis qui est pérenne.

Quant à la taille du chaume, elle varie d'une espèce à l'autre. Chez certaines espèces telles que $D$. aristulata et $D$. patagiata, le chaume est très court et ne dépasse guère 25 cm. D'autres espèces comme $D$. acuminatissima, D. argillacea, D. ciliaris, $D$. debilis, $D$. delicatula, $D$. exilis, $D$. gayana, $D$. gentilis, D. horizontalis, D. leptorhachis, D. longiflora, D. nuda, D. ternata, D. sanguinalis et $D$. velutina présentent un chaume atteignant $50 \mathrm{~cm}$ de hauteur mais sans dépasser $120 \mathrm{~cm}$. Le troisième groupe est formé de grandes herbes de hauteur égale ou supérieure à 200 $\mathrm{cm}$. C'est le cas de $D$. diagonalis et $D$. perrottetii.

Le port du chaume est constamment dressé. Toutefois, celui-ci peut présenter ou non des ramifications. En effet, le chaume est simple, parfois ramifié chez $D$. argillacea, $D$. exilis, D. gayana et D. horizontalis. Digitaria diagonalis se distingue par son chaume simple, non ramifié alors que chez les autres espèces étudiées, les chaumes sont toujours ramifiés.

Concernant la pubescence des nœuds, elle est variable et permet de distinguer trois groupes :

- les espèces à nœuds glabres: $D$. acuminatissima, $D$. diagonalis, D. exilis, $D$. gayana, D. horizontalis, D. longiflora, $D$. perrottetii, D. ternata et D. velutina ;

- les espèces à nœuds parfois glabres, parfois pubescents: D. gentilis, D. leptorhachis, D. sanguinalis et ;

- les espèces à nœuds pubescents: $D$. argillacea, D. aristulata, D. ciliaris, $D$. debilis, D. delicatula, D. nuda et D. patagiata.

Pour l'ensemble des espèces du genre Digitaria, les entre-nœuds des chaumes sont glabres sauf chez $D$. longiflora où ils sont souvent ciliés sous l'inflorescence.

\section{La feuille}

L'observation de la feuille des différentes espèces de Digitaria présentes au Sénégal a permis d'obtenir les résultats consignés dans le Tableau 2. Ces résultats montrent que les caractères de la feuille et notamment de la gaine, la ligule et le limbe, sont variables suivant les espèces.

\section{- La gaine}

En ce qui concerne la pubescence de la gaine, 3 groupes sont formés :

- $\quad$ soit la gaine est glabre : c'est le cas de D. exilis, D. perrottetii et D. ternata ; 
- $\quad$ soit elle est pubescente: $D$. argillacea, D. aristulata, D. diagonalis, $D$. leptorhachis, D. nuda, D. patagiata et $D$. velutina;

- $\quad$ soit elle est glabre mais parfois pubescente: D. acuminatissima, D. ciliaris, $D$. debilis, D. delicatula, D. gayana, $D$. gentilis, D. horizontalis, D. longiflora et $D$. sanguinalis.

\section{- La ligule}

La ligule est membraneuse et tronquée sauf chez D. leptorhachis où le sommet est arrondi. Sa longueur varie suivant les espèces. En effet, elle est très courte $(0,5 \mathrm{~mm}$ environ) chez D. argillacea, D. aristulata, D. ciliaris, $D$. delicatula, D. diagonalis et D. gayana. $\mathrm{Sa}$ longueur est moyenne et comprise entre 1 et 2 $\mathrm{mm}$ chez la plupart des espèces (D. acuminatissima, D. debilis, D. exilis, $D$. gentilis, D. horizontalis, D. leptorhachis, D. longiflora, D. nuda, D. perrottetii et $D$. velutina. Les espèces telles que $D$. patagiata, $D$. ternata et $D$. sanguinalis se reconnaissent par leur ligule plus développée et dont la longueur est $\square 2 \mathrm{~mm}$. Cette ligule est le plus souvent glabre mais elle peut être parfois pubescente (D. acuminatissima, D. argillacea, D. aristulata, D. delicatula, D. diagonalis et D. gayana).

\section{- Le limbe}

Les dimensions du limbe diffèrent considérablement au sein d'une même espèce et entre espèces. Toutefois, il est important de noter que chez $D$. aristulata, la longueur du limbe est très courte et ne dépasse pas $3 \mathrm{~cm}$ alors que chez $D$. diagonalis, cette longueur atteint $60 \mathrm{~cm}$. Parallèlement, la largeur du limbe est aussi très hétérogène. Cependant, $D$. aristulata est reconnaissable par son limbe ne dépassant pas $3 \mathrm{~mm}$ de large contrairement à $D$. diagonalis, D. horizontalis et $D$. perrottetii dont le limbe est supérieur à $10 \mathrm{~mm}$ de large.

Le limbe est généralement linéaire. Le sommet du limbe est atténué en une pointe fine sauf chez $D$. exilis où il est acuminé. $\mathrm{Sa}$ base est arrondie mais elle est parfois subcordée chez $D$. perrottetii.

Selon la présence ou non de pilosité sur le limbe, il y a :
- des espèces à limbes entièrement glabres; c'est le cas de D. exilis ;

- des espèces à limbes glabres mais pouvant être parfois pubescents; c'est le cas de $D$. acuminatissima, $D$. argillacea, $D$. ciliaris, D. debilis, D. delicatula, D. diagonalis, D. gentilis, D. horizontalis, D. longiflora, D. ternata et ;

- des espèces à limbes toujours pubescents comme D. aristulata, D. gayana, D. leptorhachis, D. nuda, D. patagiata, D. perrottetii, $D$. sanguinalis et $D$. velutina.

S'agissant de la marge du limbe, celleci est habituellement cartilagineuse. Outre, sa surface est généralement scabre sauf chez $D$. debilis où elle est lisse. Cette marge est le plus souvent glabre à l'exception des espèces $D$. gayana et D. leptorhachis où elle est pubescente.

\section{L’inflorescence générale}

L'étude des caractères de l'inflorescence générale des différentes espèces du genre Digitaria a conduit aux résultats résumés dans le Tableau 3.

De ce tableau, il ressort que l'inflorescence générale de Digitaria est formée d'une ou de plusieurs racèmes spiciformes. La très grande variabilité de leurs nombres au sein et entre espèces n'autorise pas une séparation en groupes bien distincts. Cependant, le nombre de racèmes de certaines espèces est caractéristique. C'est le cas de $D$. patagiata dont l'inflorescence est constituée d'un seul racème et de $D$. perrottetii à $\mathrm{n}$ racèmes.

Suivant l'insertion des racèmes sur l'axe de l'inflorescence, plusieurs types d'organisation sont observés :

- un seul racème chez D. patagiata ;

- les racèmes uniquement digités chez D. argillacea, D. aristulata, D. delicatula, D. exilis, D. longiflora, D. nuda et D. ternata;

- les racèmes digités ou parfois disposés le long de l'axe principal chez $D$. acuminatissima et D. gentilis ;

- les racèmes digités ou subdigités chez D. ciliaris, D. gayana, D. horizontalis, $D$. sanguinalis et $D$. velutina ; 
- les racèmes subdigités chez $D$. debilis et D. leptorhachis ;

- les racèmes disposés le long de l'axe principal chez $D$. diagonalis et ;

- les racèmes verticillés et disposés le long de l'axe principal chez $D$. perrottetii.

$\mathrm{La}$ longueur des racèmes varie constamment. Toutefois, il est à noter que les racèmes atteignent $20 \mathrm{~cm}$ ou plus chez $D$. acuminatissima, D. ciliaris, D. diagonalis et D. nuda. Quant au rachis des racèmes, il est triquètre et non ailé sauf chez $D$. acuminatissima, D. aristulata, D. gayana, D. gentilis, D. nuda, D. ternata, D. sanguinalis et $D$. velutina où il est triquètre ailé. Pour toutes les espèces étudiées, la marge du rachis est régulièrement scabre.

\section{L'épillet}

L'observation de l'épillet des différentes espèces de Digitaria présentes au Sénégal a permis d'obtenir les résultats consignés dans le Tableau 4. Ces résultats montrent que les caractéristiques de l'épillet sont très variables suivant les espèces.

En ce qui concerne la disposition des épillets, il apparaît que :

- certaines espèces présentent des épillets toujours regroupés soit par deux chez la plupart des espèces (D. acuminatissima, $D$. ciliaris, D. debilis, D. gentilis, D. horizontalis, D. leptorhachis, D. perrottetii, D. sanguinalis, $D$. velutina) ou par trois (D. delicatula et $D$. longiflora) ;

- d'autres espèces se caractérisent par un regroupement variable des épillets. Il s'agit de D. patagiata (solitaire ou par 2); $D$. argillacea, D. aristulata, D. exilis (par 2 ou 3) ; D. gayana, D. ternata (par 3 ou 4) et $D$. diagonalis (2, 3 ou même jusqu'à 6).

La longueur des épillets est très hétérogène. Les épillets sont longs de 1,4 à 4,5 $\mathrm{mm}$ suivant les espèces. Leur forme est notamment très variable mais la plupart des espèces présentent des épillets elliptiques. Néanmoins, les épillets peuvent parfois être lancéolés (D. aristulata, $D$. debilis, $D$. gentilis, D. horizontalis), lancéolés-oblongs
(D. patagiata, D. velutina) ou encore ovales (D. gayana).

Le sommet des épillets est généralement aigu. Toutefois, il peut être acuminé (D. acuminatissima, Digitaria aristulata, D. debilis), subacuminé (D. exilis, D. gayana), ou aigu-acuminé (D. ciliaris). D. ternata se reconnaît par ses épillets à sommet obtus à subobtus alors que $D$. delicatula présente des épillets à sommet aigu, enflé en massue et parfois brièvement mucroné.

Les épillets peuvent comporter des poils ou non. Ils sont glabres chez $D$. diagonalis et $D$. exilis. $\mathrm{Si}$ les poils sont présents, ceux-ci sont de différentes natures. Il y a ainsi :

- des poils apprimés pour une grande partie des espèces (D. acuminatissima, D. ciliaris, D. debilis, D. gentilis, D. horizontalis, D. perrottetii, D. sanguinalis, D. velutina);

- des poils soyeux, rigides chez $D$. argillacea, souples à sommet enflé en massue chez D. delicatula et D. ternata, longs, argentés ou rouges chez D. gayana et argenté chez D. leptorhachis ;

- des poils apprimés mais soyeux sur les marges de l'épillet chez D. nuda et ;

- des poils courts verruqueux caractéristiques chez D. aristulata, $D$. longiflora et D. patagiata.

L'une des particularités des épillets du genre Digitaria est la dimension des pédicelles. En effet, les pédicelles des épillets successifs sont inégaux. La surface des pédicelles est habituellement scabre sauf chez $D$. aristulata, D. exilis et D. longiflora où elle est lisse.

L'observation de la présence ou non de poils à la surface des pédicelles montre, en général, que la plupart des espèces ont des pédicelles glabres. Exceptés, d'une part $D$. diagonalis et $D$. ternata à pédicelles pubescents et, d'autre part, D. exilis et $D$. nuda à pédicelles glabres parfois pubescents. Parmi ces quatre espèces, D. diagonalis se reconnaît par ses pédicelles munis de poils aussi longs ou dépassant le sommet de l'épillet. 


\section{Les glumes}

La morphologie de l'épillet, plus particulièrement celle de ces glumes, est une des caractéristiques fondamentales de la reconnaissance du genre Digitaria. En effet, Digitaria se distingue des autres genres de par sa glume inférieure absente ou très réduite alors que la glume supérieure est bien développée. Le Tableau 5 résume les différents caractères observés sur ces deux types de glumes.

Concernant la glume inférieure, elle est toujours absente chez $D$. argillacea, $D$. delicatula, D. diagonalis, D. perrottetii, $D$. ternata. Dans certains cas, elle aussi absente chez D. aristulata, D. debilis, D. gentilis, D. horizontalis, D. longiflora, $D$. nuda et $D$. sanguinalis et $D$. velutina. Lorsque cette glume est présente, elle peut revêtir plusieurs formes :

- $\quad$ en écaille chez D. acuminatissima, $D$. debilis et D. patagiata ;

- en bourrelet chez D. aristulata, D. exilis, D. gentilis, D. horizontalis, D. nuda, D. sanguinalis et $D$. velutina ;

- $\quad$ en anneau chez D. leptorrachis ou ;

- $\quad$ en une minuscule membrane chez $D$. longiflora.

Le sommet de la glume inférieure est souvent tronqué. Toutefois, chez $D$. acuminatissima et $D$. ciliaris, il est respectivement ovale et aigu.

Contrairement à la glume inférieure, la glume supérieure des Digitaria est bien développée. Du fait de leur grande variabilité, les caractères de la glume supérieure sont très discriminants et sont ainsi fréquemment utilisés pour la distinction des espèces.

Les différentes formes de la glume supérieure témoignent de ce qualificatif qui lui est réservé. En effet, la glume supérieure est soit :

- elliptique: D. gentilis, D. leptorhachis, D. longiflora, D. perrottetii ;

- lancéolée : D. argillacea, D. debilis, D. sanguinalis, D. velutina ;

- $\quad$ ovale : D. diagonalis, D. patagiata ;

- oblongue : D. aristulata, D. exilis ;
- linéaire-lancéolée: $D$. ciliaris, $D$. horizontalis ;

- oblongue-lancéolée : D. gayana, D. ternata;

- lancéolée-ovale : D. acuminatissima ;

- ovale-oblongue : D. delicatula ;

- oblongue-triangulaire : D. nuda.

A l'image de la forme de la glume, le sommet de celle-ci est aussi bien diversifié. Cependant, plus de la moitié des espèces ont une glume supérieure à sommet aigu. Concernant les autres espèces, le sommet est soit acuminé (D. acuminatissima, D. debilis), soit obtus (D. diagonalis, D. exilis, D. leptorhachis, D. ternata et D. sanguinalis) ou encore obtus-subaigu (D. aristulata).

Les différentes longueurs relatives de la glume supérieure permettent de classer les espèces en 3 groupes :

- les espèces à glume supérieure ne dépassant pas $1 / 2$ de la longueur de l'épillet: $D$. acuminatissima, D. aristulata, D. diagonalis, D. horizontalis, D. sanguinalis ;

- les espèces à glume supérieure de $1 / 2$ à 9/10 de la longueur de l'épillet: $D$. argillacea, $D$. ciliaris, $D$. delicatula, $D$. gayana, D. nuda, D. perrottetii, D. ternata, D. velutina et ;

- les espèces à glume supérieure aussi longue que l'épillet : $D$. debilis, D. exilis, $D$. gentilis, D. leptorhachis, D. longiflora, D. patagiata.

La glume supérieure est de façon générale 3-nervée. Cependant, elle peut être 5nervée. C'est le cas de D. leptorhachis, D. longiflora et $D$. patagiata. La glume supérieure de D. acuminatissima a 3 ou 5 nervures de même que $D$. exilis (rarement 3 ) contrairement à $D$. debilis qui en possède 5 ou 7 nervures. Exceptionnellement, la glume supérieure de $D$. diagonalis est dépourvue de nervure.

La majeure partie des espèces étudiées présentent des poils sur leurs glumes supérieures sauf chez $D$. diagonalis et $D$. exilis à glumes glabres. 


\section{Les fleurs}

A l'image des autres genres de la Tribu des Paniceae à laquelle il appartient, le genre Digitaria présente des épillets biflores. Cependant, bien que morphologiquement biflores, les épillets sont fonctionnellement uniflores. En d'autres termes, la fleur inférieure est stérile, représentée uniquement par la lemma inférieure alors que la fleur supérieure est fertile avec la présence d'une lemma et d'une paléole supérieures. Ainsi, cette spécificité de l'épillet de Digitaria, décrite précédemment, la doit beaucoup à celle de ces fleurs. Les caractères de ces dernières, consignés dans le Tableau 6 , apportent des détails supplémentaires dans l'identification des différentes espèces étudiées.

La forme de la lemma de la fleur inférieure est souvent elliptique. Toutefois, elle est oblongue chez $D$. velutina ; oblonguelancéolée chez $D$. aristulata et $D$. nuda; oblongue-elliptique chez D. ternata; lancéolée-elliptique chez $D$. delicatula; lancéolée-ovale chez $D$. diagonalis et ovaleoblongue chez $D$. sanguinalis. Le sommet de la lemma est aigu pour l'ensemble des espèces sauf D. ternata (subobtus).

Pour la quasi-totalité des espèces étudiées, la lemma de la fleur inférieure est aussi longue que l'épillet. Deux espèces seulement présentent une lemma inférieure plus courte que l'épillet. Il s'agit de $D$. aristulata ( $4 / 5$ de la longueur de l'épillet) et D. debilis (7/10 au 4/5 de la longueur de l'épillet). L'espèce $D$. diagonalis est caractérisée par une lemma inférieure pouvant être inférieure ou égale à la longueur de l'épillet (4/5 à 1 fois la longueur de l'épillet).

Pour ce qui est des nervures présentes sur la lemma, leur nombre est constant ou variable au sein d'une espèce. Il existe ainsi :

- des espèces à lemma inférieure 7nervée : $D$. acuminatissima, $D$. argillacea, $D$. ciliaris, $D$. debilis, D. horizontalis, $D$. leptorhachis, D. longiflora, D. sanguinalis et D. velutina;

- des espèces à lemma inférieure 5nervée: $D$. delicatula, $D$. gentilis et $D$. patagiata ;
- des espèces à lemma inférieure 5-7nervée : $D$. aristulata, $D$. gayana, $D$. nuda et D. perrottetii ;

- des espèces à lemma inférieure 3-57-nervée : $D$. diagonalis.

- des espèces à lemma inférieure 5-9nervée : D. ternata et ;

- des espèces à lemma inférieure 7-9nervée : D. exilis.

Comme il a été constaté au niveau de la glume supérieure, toutes les espèces étudiées présentent une lemma inférieure pubescente hormis $D$. diagonalis et $D$. exilis.

La fleur supérieure est fertile et est constituée d'une lemma et d'une paléole bien développées. Elle présente un certain nombre de caractères qui participent à la définition du genre et qui sont constants notamment sur toutes les espèces étudiées. Ces caractères sont :

- la pubescence de la lemma supérieure qui est glabre ;

- la longueur relative de la paléole, subégale à sa lemma supérieure et ;

- le nombre d'étamines égale à trois.

Les caractères discriminants concernent la forme, le sommet et la longueur relative de la lemma de la fleur supérieure. En fait, différents groupes peuvent être constitués suivant la forme de la lemma supérieure :

- les espèces à lemma supérieure elliptique : D. debilis, D. gayana, D. gentilis, $D$. leptorhachis, D. longiflora, D. patagiata, D. ternata, D. velutina;

- les espèces à lemma supérieure ovale : D. acuminatissima, $D$. argillacea, $D$. delicatula, D. diagonalis ;

- les espèces à lemma supérieure lancéolée : $D$. horizontalis, $D$. sanguinalis ;

- les espèces à lemma supérieure oblongue-lancéolée : $D$. aristulata, $D$. ciliaris, D. nuda et ;

- les espèces à lemma supérieure obovale : $D$. exilis et $D$. perrottetii.

La variabilité de la forme du sommet de la lemma supérieure permet de scinder les espèces en 2 groupes : 
- Groupe 1 : espèces dont le sommet de la lemma supérieure est uniforme ; le sommet est

- acuminé : D. acuminatissima, D. aristulata, D. debilis, D. longiflora et $D$. sanguinalis;

- aigu: D. argillacea, D. ciliaris, D. gentilis, D. horizontalis, D. leptorhachis, et $D$. velutina;

- ou obtus : D. diagonalis.

- Groupe 2 : espèces dont le sommet de la lemma supérieure est variable (6 cas); le sommet est aigu-mucroné (D. delicatula), obtus-arrondi (D. exilis), acuminé-rostré (D. gayana), aigu-acuminé (D. nuda) ou obtusmucroné (D. perrottetii).

Le troisième et dernier caractère discriminant de la lemma supérieure concerne sa longueur relative. La lemma supérieure est :

- inférieure à la longueur de l'épillet : D. acuminatissima, D. aristulata, D. delicatula, D. gentilis, D. nuda, D. patagiata, D. perrottetii, D. ternata;

- inférieure ou égale à la longueur de l'épillet : D. sanguinalis, D. velutina ;

- $\quad$ égale à la longueur de l'épillet: $D$. argillacea, D. ciliaris, $D$. debilis, $D$. diagonalis, $D$. exilis, $D$. gayana, $D$. horizontalis, D. leptorhachis et D. longiflora.

\section{Le fruit}

Les résultats consignés dans le Tableau 7 montrent que les caractères morphologiques $\mathrm{du}$ fruit peuvent apporter des informations non négligeables dans la reconnaissance des espèces.
Le fruit de Digitaria, comme celui de toutes les autres Graminées, est un caryopse mais de formes diverses. Ainsi, ce fruit peut être elliptique $(D$. delicatula, $D$. diagonalis, D. gentilis, D. leptorhachis, D. longiflora, D. perrottetii, D. ternata), oblong (D. aristulata, $D$. ciliaris, D. horizontalis, D. nuda, D. velutina), oblong-lancéolé (D. argillacea, $D$. patagiata), ovée-oblong (D. debilis, D. gayana), lancéolé ( $D$. sanguinalis), linéaireoblong ( $D$. acuminatissima) ou ovoïde ( $D$. exilis). A l'exception de D. gayana (à sommet rostré) et $D$. horizontalis (à sommet aiguacuminé), le sommet du fruit est aigu pour le reste des espèces. Sa longueur est également variable et, pour la plupart des cas, elle ne dépasse pas $1,5 \mathrm{~mm}$. Cependant, chez $D$. argillacea, D. delicatula, D. horizontalis, D. patagiata et $D$. ternata, cette longueur est comprise entre 1,5 et $2 \mathrm{~mm}$. Les espèces telles que $D$. acuminatissima et $D$. ciliaris sont caractérisées par un fruit dépassant $2 \mathrm{~mm}$ de long. Enfin, la couleur du fruit (grain) permet de distinguer plus facilement certaines espèces. C'est le cas, par exemple, de $D$. delicatula caractérisée par son grain noirâtre, de $D$. acuminatissima et $D$. aristulata, à grains blanchâtres. Les autres espèces ont des grains colorés: beige chez D. gentilis, $D$. horizontalis; brun chez D. ciliaris, $D$. perrottetii, D. sanguinalis, D. velutina; brun à noir chez $D$. argillacea, $D$. diagonalis, $D$. ternata; brun ou gris chez $D$. longiflora, $D$. nuda; gris pourpré chez D. debilis, $D$. leptorhachis, D. patagiata; jaunâtre ou brun chez D. gayana. Quant au grain de D. exilis, il présente différentes couleurs (blanc, jaune ou pourpre) suivant les variétés.

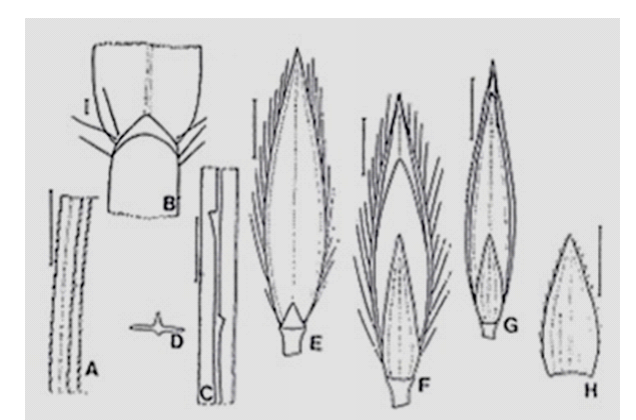

Figure 1 : Digitaria acuminatissima. A. Marge d'un limbe foliaire (fragment). B. Base du limbe foliaire, ligule et partie supérieure de la gaine étalée. C. Axe d'un épi (fragment). D. Id., en coupe transversale. E. Epillet, avec la glume 
inférieure et la lemma de la fleur inférieure. F. Face opposée de l'épillet, avec la glume supérieure et la lemma de la fleur supérieure. G. Id. H. Glume supérieure. A-F et H : LISOWSKI B 1845 (Tchad) ; G : BERHAUT 616. (Vanden Berghen, 1991).

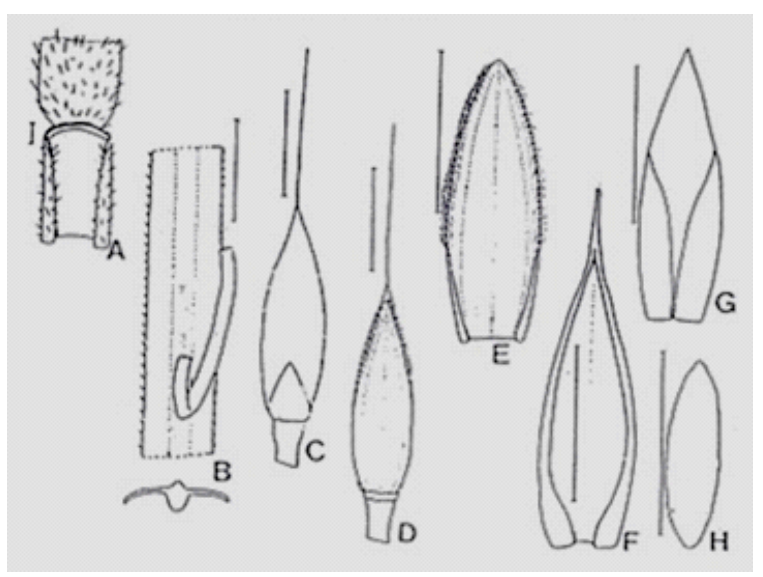

Figure 2 : Digitaria aristulata. A. Base du limbe foliaire, ligule et partie supérieure de la gaine. B. Axe d'un racème (fragment) ; coupe transversale dans cet axe. C. Epillet, avec la glume supérieure et la lemma de la fleur supérieure. D. Face opposée de l'épillet, avec la lemma de la fleur inférieure. E. Lemma de la fleur inférieure. F. Lemma de la fleur supérieure G. Paléole de la fleur supérieure. H. Caryopse. A-H : A. CHEVALIER 1094 (Mali). (Vanden Berghen, 1991).

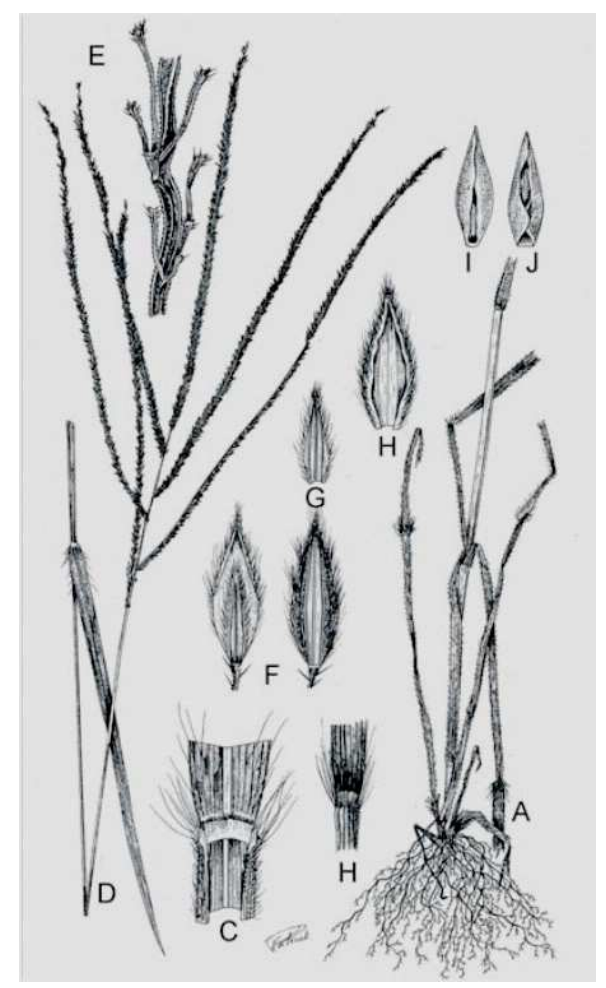

Figure 3 : Digitaria argillacea. A. Base de la plante. B. Noeud. C. Ligule. D. Inflorescence. E. Fragment de rachis d'un racème. F. Epillet en faces dorsale et ventrale. G. Glume supèrieure. H. Lemma de la fleur inférieure. I. Fleur supérieure. J. Paléoles de la fleur supérieure (Poilecot). 


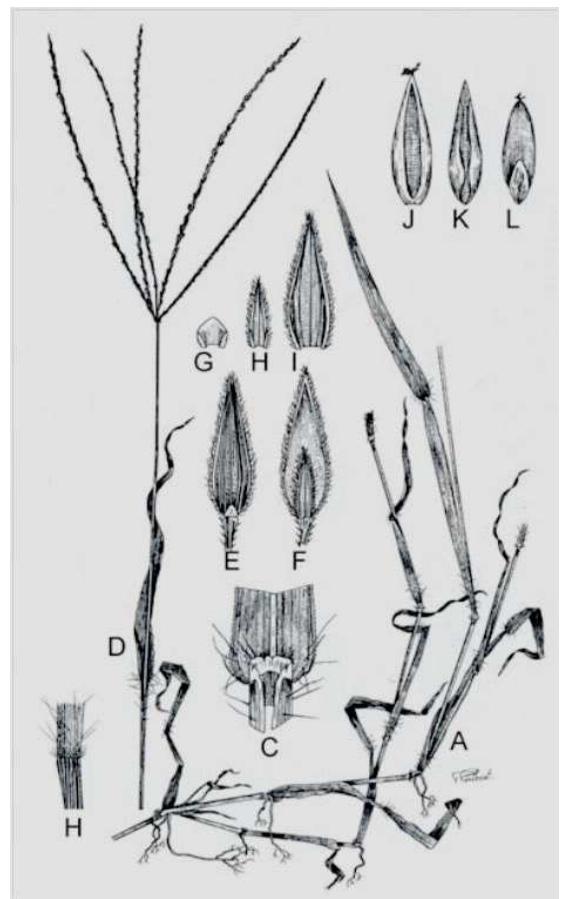

Figure 4 : Digitaria ciliaris. A. Base de la plante. B. Noeud. C. Ligule. D. Inflorescence. E et F. Epillet en faces dorsale et ventrale. G et H. Glumes inférieure et supérieure. I. Lemma de la fleur inférieure. J. Fleur supérieure. K. Paléole de la fleur supérieure. L. Caryopse (Poilecot).

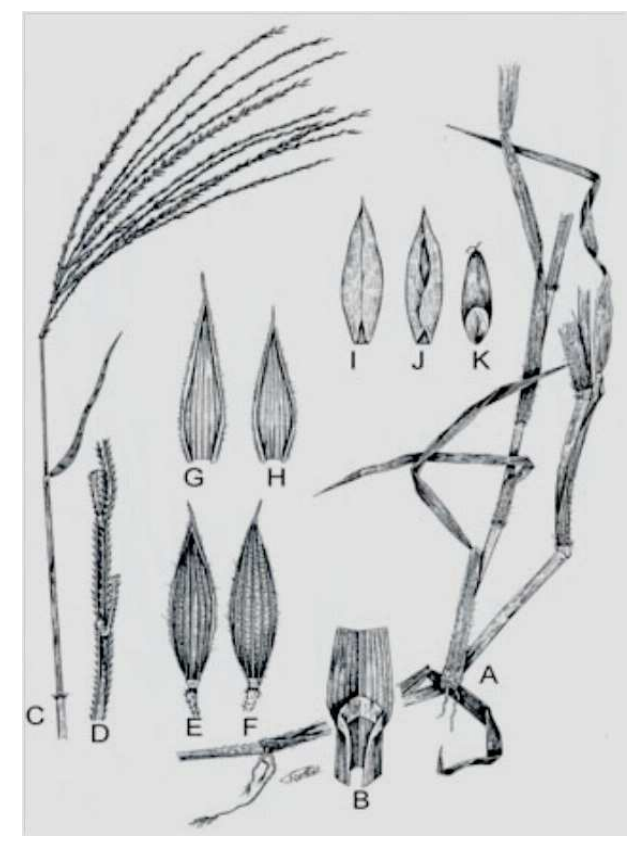

Figure 5 : Digitaria debilis. A Base de la plante. B. Ligule. C. Inflorescence. D. Fragment de rachis d'un racème et pédicelles. E et F. Epillet en faces dorsale et ventrale. G. Glume supérieure. H. Lemma de la fleur inférieure. I. Fleur supérieure. J. Paléole de la fleur supérieure. K. Caryopse (Poilecot). 


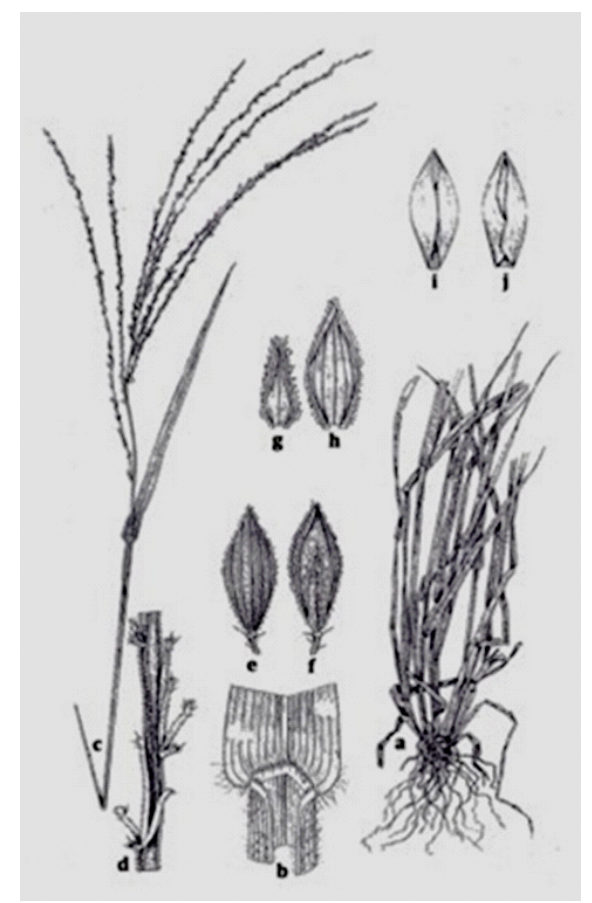

Figure 6 : Digitaria delicaluta. A Base de la plante. B. Ligule. C. Inflorescence. D. Fragment de rachis d'un racème et pédicelles. E et F. Epillet en faces dorsale et ventrale. G. Glume supérieure. H. Lemma de la fleur inférieure. I. Fleur supérieure. J. Paléole de la fleur supérieure. (Poilecot).

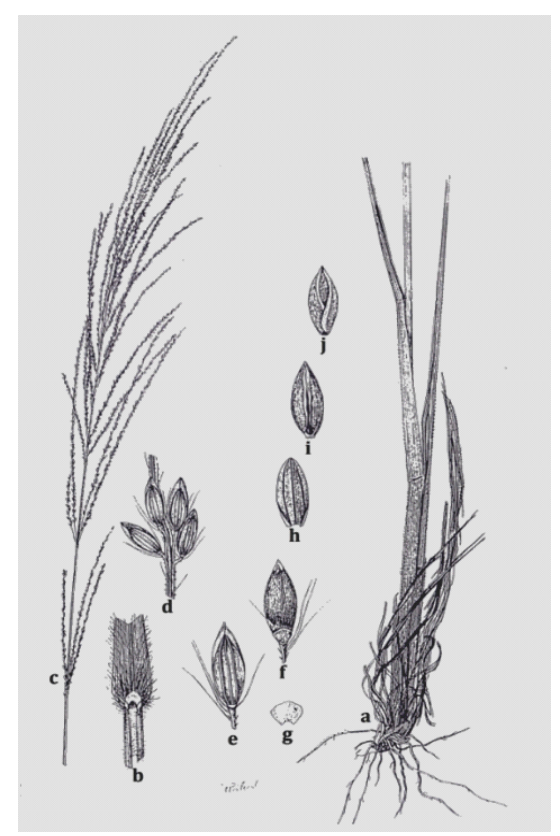

Figure 7 : Digitaria diagonalis. a. Base de la plante. b. Ligule. c. Inflorescence. d. Fragment de racème. e et f. Epillet en faces dorsale et ventrale. g. Glume supérieure. h. Lemma de la fleur inférieure. i. Fleur supérieure. j. Paléole de la fleur supérieure. (Poilecot). 


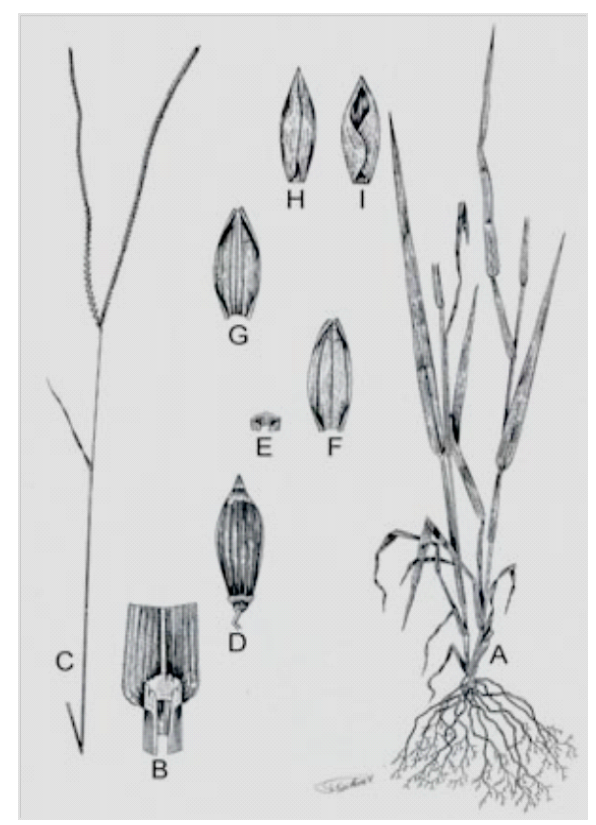

Figure 8 : Digitaria exilis. A. Base de la plante. B. Ligule. C. Inflorescence. D. Epillet. E et F. Glumes inférieure et supérieure. G. Lemma de la fleur inférieure. H. Fleur supérieure. I. Paléole de la fleur supérieure (Poilecot).

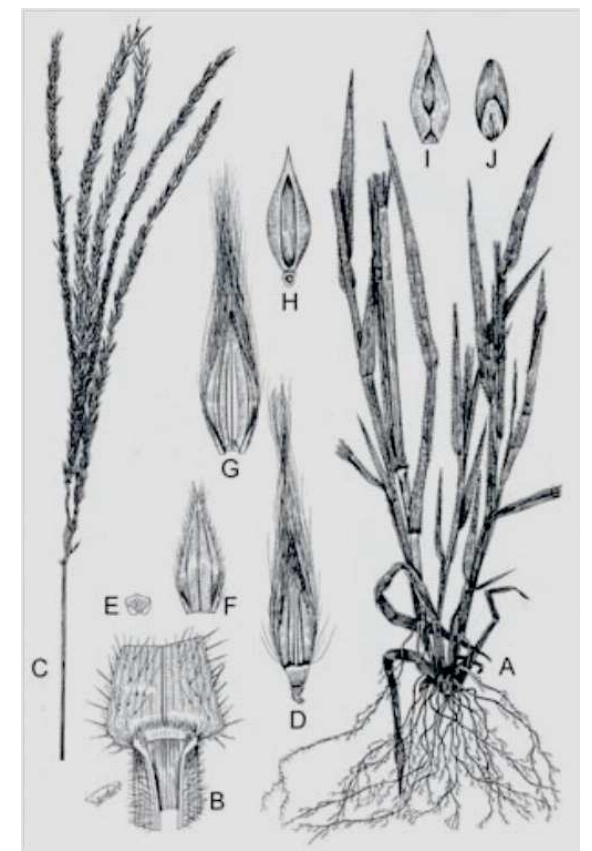

Figure 9 : Digitaria gayana. A. Base de la plante. B. Ligule. C. Inflorescence. D. Epillet. E et F. Glumes inférieure et supérieure. G. Lemma de la fleur inférieure. H. Fleur supérieure. I. Paléole de la fleur inférieure. J. Caryopse (Poilecot). 


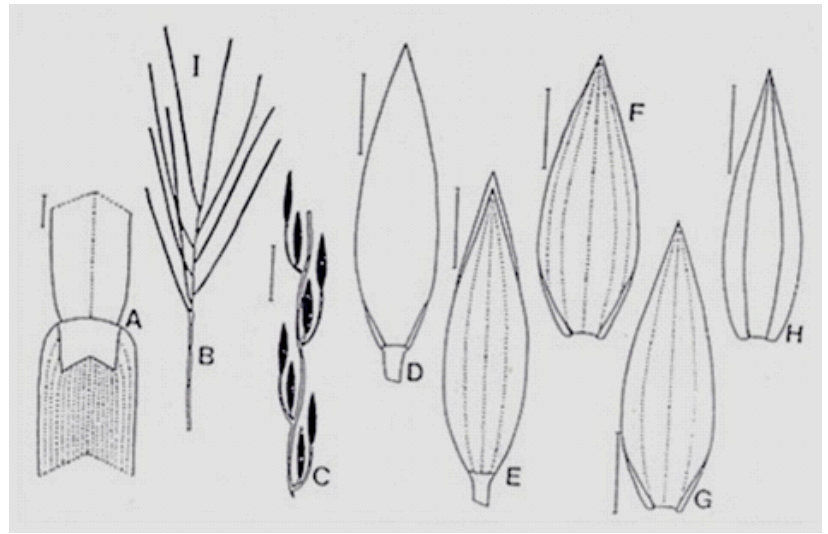

Figure 10 : Digitaria gentilis. A. Base du limbe foliaire, ligule soudée latéralement aux oreillettes de la gaine ; partie supérieure de la gaine, étalée. B. Inflorescence, schématisée. C. Fragment de racème. D. Epillet, avec la lemma de la fleur inférieure. E. Face opposée de cet épillet, avec la glume supérieure. F. Lemma de la fleur inférieure G. Glume supérieure. H. Lemma de la fleur supérieure. A-H : TROCHAIN 477 bis : P. (Vanden Berghen, 1991).

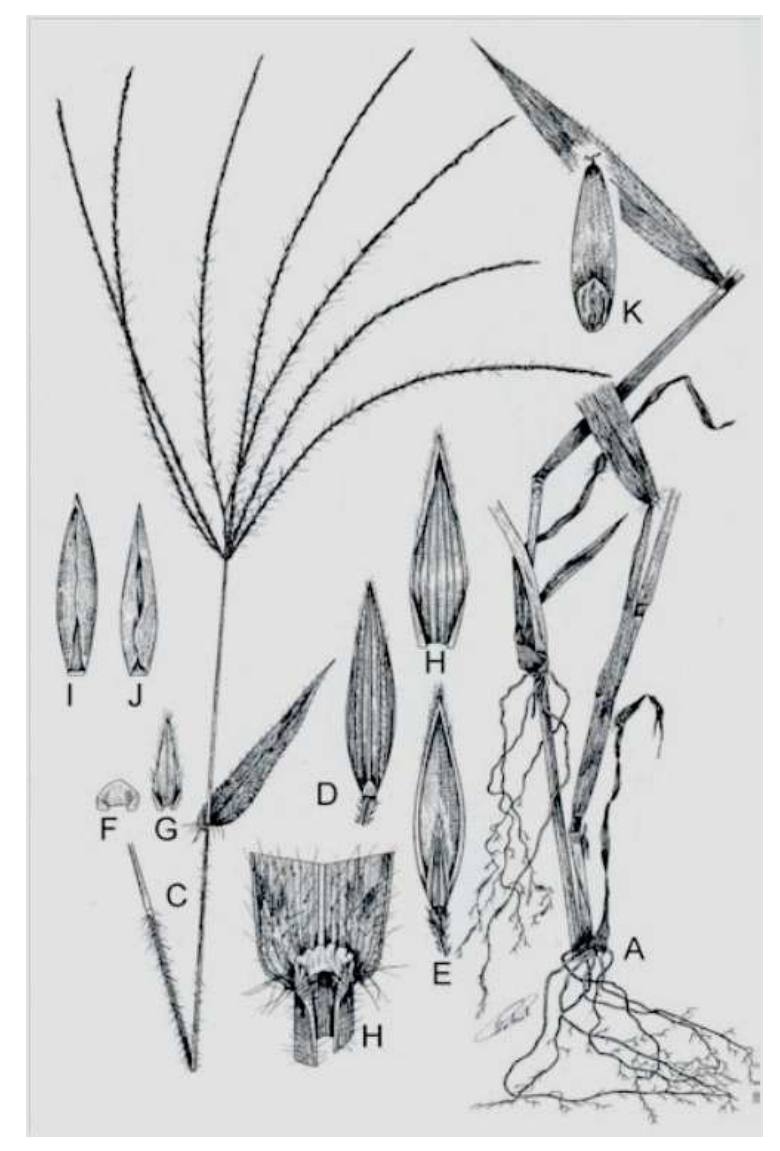

Figure 11 : Digitaria horizontalis. A. Base de la plante. B. Ligule. D et E. Epillet en faces dorsale et ventrale. F et G. Glume inférieure et supérieure. H. Lemma de la fleur inférieure. I. Fleur supérieure. J. Paléole de la fleur supérieure. K. Caryopse (Poilecot). 


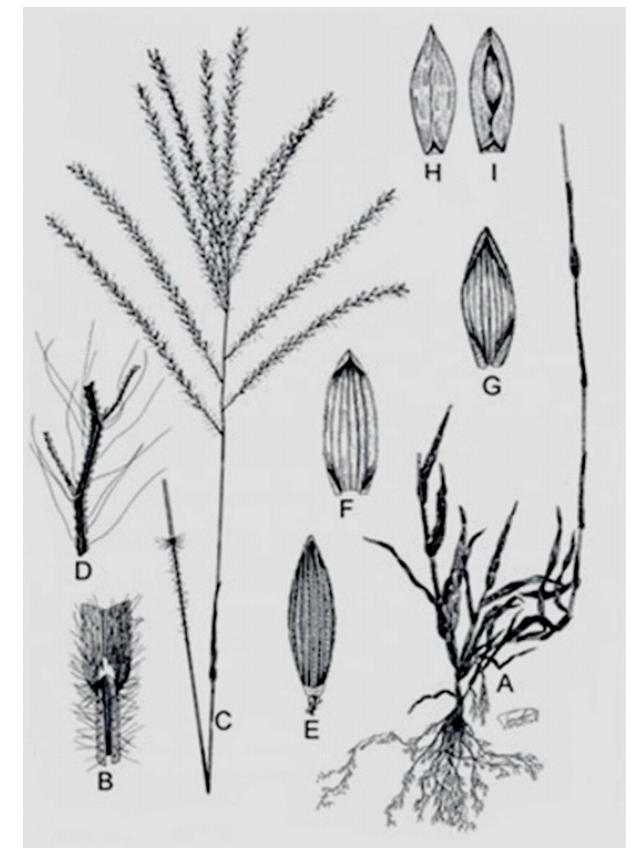

Figure 12 : Digitaria leptorhachis. A. Base de la plante. B. Ligule. C. Inflorescence. D. Fragment de rachis d'une racine et pédicelles. E. Epillet en face ventrale. F. Glume supérieure. G. Lemma de la fleur inférieure. H. Fleur supérieure. I. Paléole de la fleur supérieure (Poilecot).

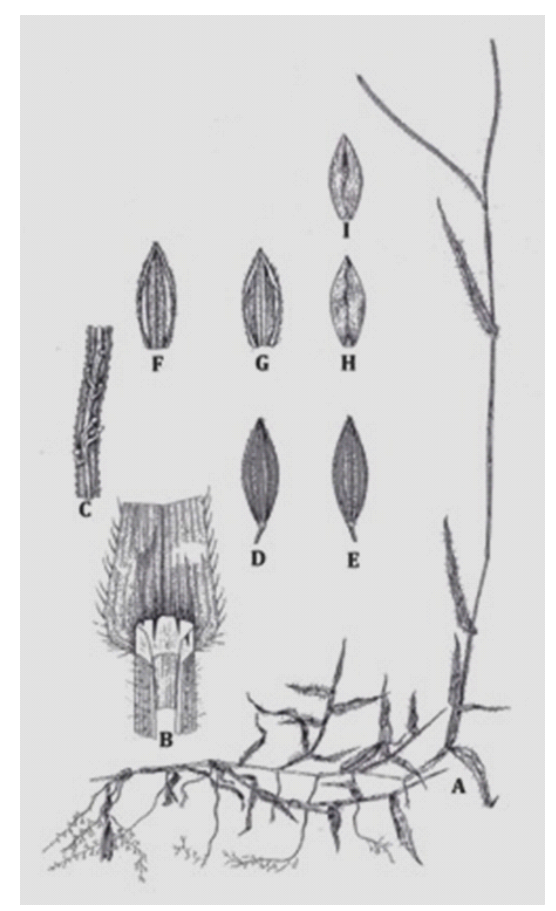

Figure 13 : Digitaria longiflora. A. Port de la plante. B. Ligule. C. Fragment de rachis avec les pédicelles. D et E. Epillet en face dorsale et ventrale. F. Glume supérieure. G. Lemma de la fleur inférieure. H. Fleur supérieure. I. Paléole de la fleur supérieure (Poilecot). 


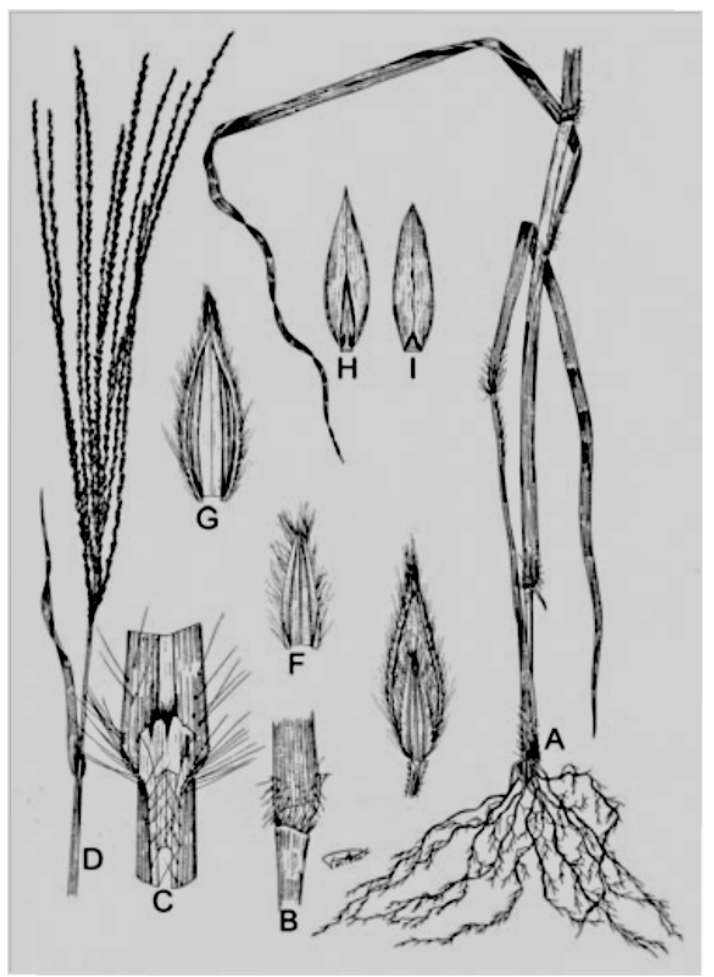

Figure 14 : Digitaria nuda. A. Base de la plante. B. Noeud. C. Ligule. D. Inflorescence. E. Epillet. F. Glume supérieure. G. Lemma de la fleur inférieure. H. Fleur supérieure. I. Paléole de la fleur supérieure (Poilecot).

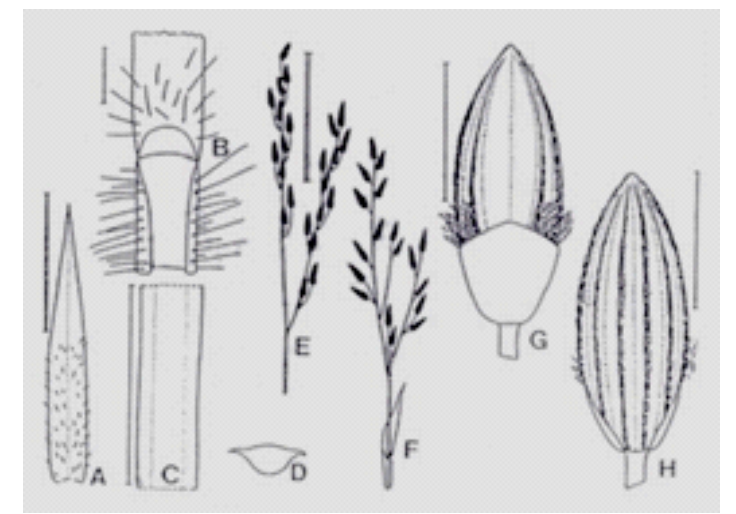

Figure 15 : Digitaria patagiata. A. Limbe foliaire. B. Base du limbe foliaire, ligule et partie supérieure de la gaine. C. Fragment de l'axe d'un racème. D. Coupe transversale de cet axe, large de 0,3 $\mathrm{mm}$ environ. E et $\mathrm{F}$ : Inflorescences, schématisées. G. Epillet, avec la glume inférieure et la lemma de la fleur inférieure. H. Face opposée de cet épillet, avec la glume supérieure. A-H : 5982. (Vanden Berghen, 1991). 


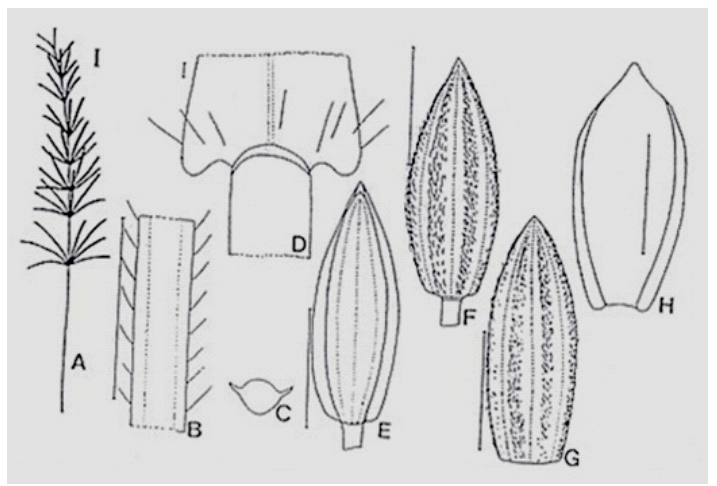

Figure 16 : Digitaria perrottetii. A. Inflorescence, schématisée. B. Fragment de l'axe d'un racème. C. Coupe transversale dans cet axe, large de $0,3 \mathrm{~mm}$ environ. D. Base du limbe foliaire, ligule et partie supérieure de la gaine, étalée. E. Epillet, avec la glume supérieure (les cils ne sont pas représentés). F. Epillet, avec la lemma de la fleur inférieure. G. Glume supérieure. H. Lemma de la fleur supérieure. A-H : BERHAUT 419. (Vanden Berghen, 1991).

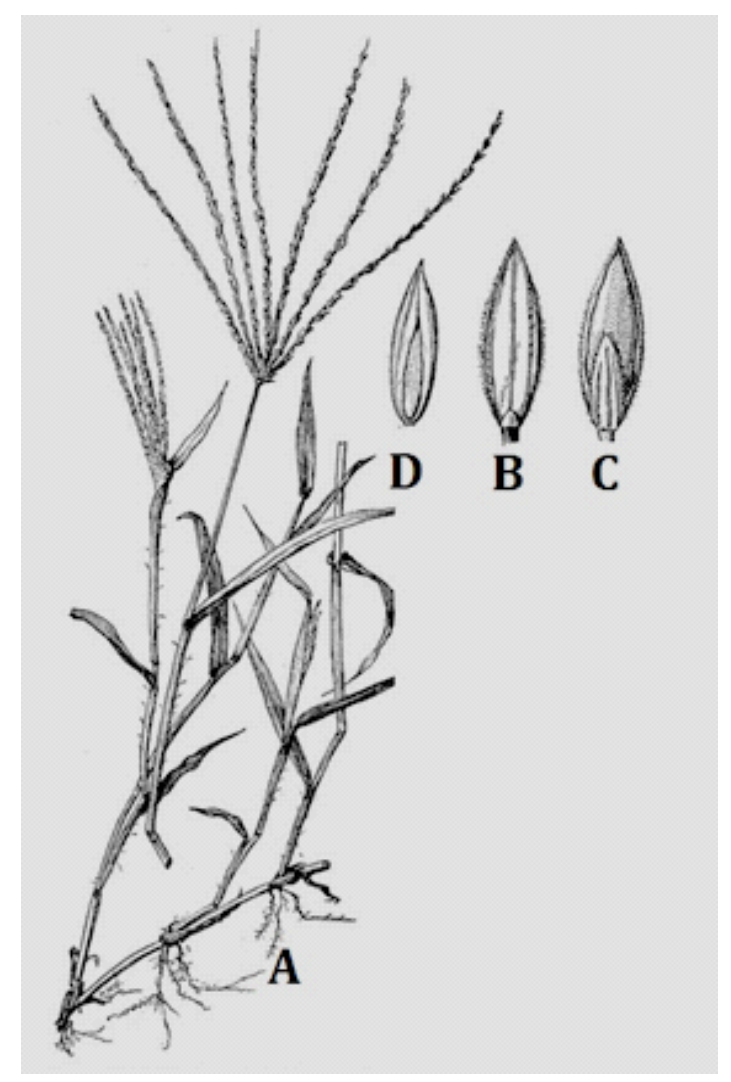

Figure 17 : Digitaria sanguinalis. A. Port de la plante X1/2. B. Epillet, avec la glume inférieure et la lemma de la fleur inférieure. C. Face opposée de l'épillet, avec la glume supérieure et la lemma de la fleur supérieure X10. D. Floret X10. (Norton 566, Kans.). 


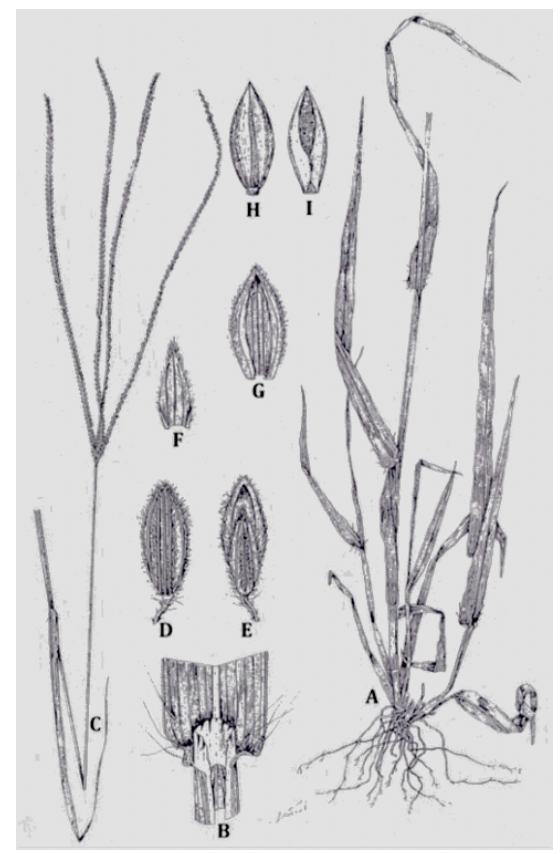

Figure 18 : Digitaria ternata. A. Base de la plante. B. Ligule. C. Inflorescence. D et E. Epillet en face dorsale et ventrale. F. Glume supérieure. G. Lemma de la fleur inférieure. H. Fleur supérieure. I. Paléole de la fleur supérieure (dessin Poilecot).

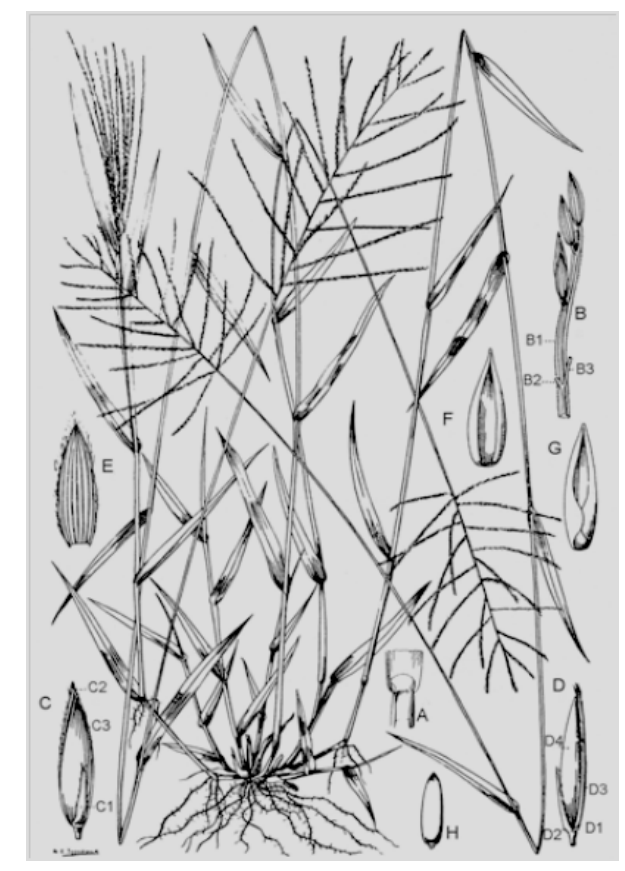

Figure 19 : Digitaria velutina. A. Ligule. B. Partie du racème. B1. Axe du racème. B2 et B3. Pédicelles des épillets. C. Epillet, vue de face. C1. Glume supérieure. C2. Lemma inférieure. C3. Lemma supérieure. D. Epillet, vue de côté. D1. Glume inférieure. D2. Glume supérieure. D3. Glume inférieure. D4. Lemma supérieure. E. Lemma inférieure. F. Lemma supérieure. G. Paléa. H. Grain (FWTA). 
A. NGOM et al. / Int. J. Biol. Chem. Sci. 10(1): 58-86, 2016

Tableau 1 : Caractères morphologiques du chaume des différentes espèces de Digitaria étudiées.

\begin{tabular}{|c|c|c|c|c|c|c|c|c|c|c|c|c|c|c|c|c|c|c|c|c|}
\hline \multirow{2}{*}{\multicolumn{2}{|c|}{ Caractères }} & \multicolumn{19}{|c|}{ Espèces } \\
\hline & & 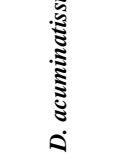 & 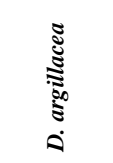 & 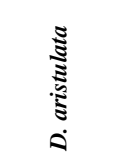 & $\begin{array}{l}\tilde{E} \\
\dot{E} \\
\dot{0}\end{array}$ & 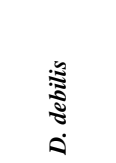 & 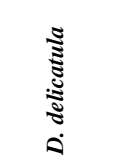 & 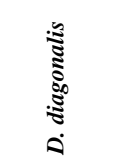 & 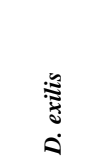 & 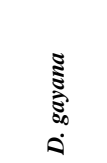 & 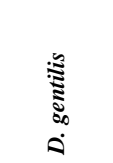 & 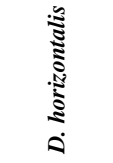 & 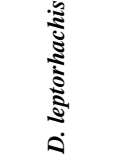 & 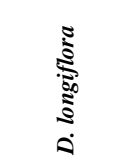 & $\stackrel{\Xi}{\vdots}$ & 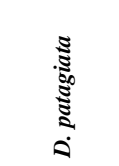 & 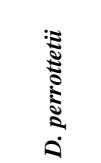 & $\frac{\Xi}{5}$ & 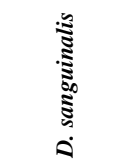 & 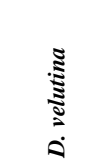 \\
\hline Cycle & biologique & annuelle & annuelle & annuelle & annuelle & annuelle & annuelle & pérenne & annuelle & annuelle & annuelle & annuelle & annuelle & annuelle & annuelle & annuelle & annuelle & annuelle & annuelle & annuelle \\
\hline Haute & $\operatorname{ur}(\mathbf{c m})$ & $30-120$ & 120 & $10-25$ & $20-100$ & $20-60$ & $50-90$ & $100-300$ & $30-80$ & $30-90$ & 80 & $20-100$ & $30-100$ & $\begin{array}{l}10-60 \\
\end{array}$ & $15-100$ & $5-25$ & $30-200$ & $20-100$ & $\begin{array}{l}10-80 \\
\end{array}$ & $20-50$ \\
\hline & Allure & dressés & dressés & dressés & dressés & dressés & dressés & dressés & dressés & dressés & dressés & dressés & dressés & dressés & dressés & dressés & dressés & dressés & dressés & dressés \\
\hline $\begin{array}{l}\mathbf{P} \\
\mathbf{O} \\
\mathbf{R} \\
\mathbf{T}\end{array}$ & $\begin{array}{l}\text { Ramificat } \\
\text { ion }\end{array}$ & ramifiés & $\begin{array}{l}\text { simples } \\
\text { ou } \\
\text { ramifiés }\end{array}$ & ramifiés & ramifiés & ramifiés & ramifiés & $\begin{array}{l}\text { simples, } \\
\text { non } \\
\text { ramifiés }\end{array}$ & $\begin{array}{l}\text { simples } \\
\text { ou } \\
\text { ramifiés }\end{array}$ & $\begin{array}{l}\text { simples } \\
\text { ou } \\
\text { ramifiés }\end{array}$ & ramifiés & $\begin{array}{c}\text { simples } \\
\text { ou } \\
\text { ramifiés }\end{array}$ & ramifiés & ramifiés & ramifiés & ramifiés & ramifiés & ramifiés & ramifiés & ramifiés \\
\hline $\begin{array}{l}\mathbf{P} \\
\mathbf{U} \\
\text { B } \\
\text { E }\end{array}$ & Nœuds & glabres & $\begin{array}{l}\text { pubescen } \\
\text { ts }\end{array}$ & $\begin{array}{l}\text { pubescen } \\
\text { ts }\end{array}$ & $\begin{array}{l}\text { pubescen } \\
\text { ts }\end{array}$ & $\begin{array}{l}\text { pubescen } \\
\text { ts }\end{array}$ & $\begin{array}{l}\text { pubescen } \\
\text { ts }\end{array}$ & glabres & glabres & glabres & $\begin{array}{c}\text { glabres } \\
\text { ou } \\
\text { pubescen } \\
\text { ts }\end{array}$ & glabres & $\begin{array}{l}\text { pubescen } \\
\text { ts ou } \\
\text { glabres }\end{array}$ & glabres & $\begin{array}{c}\text { pubescent } \\
\mathrm{s}\end{array}$ & $\begin{array}{c}\text { pubescent } \\
\mathrm{s}\end{array}$ & glabres & glabres & $\begin{array}{l}\text { glabres ou } \\
\text { pubescents }\end{array}$ & glabres \\
\hline $\begin{array}{l}\mathbf{S} \\
\mathbf{C} \\
\mathbf{E} \\
\mathbf{N} \\
\mathbf{C} \\
\mathbf{E}\end{array}$ & $\begin{array}{l}\text { Entre- } \\
\text { nœeuds }\end{array}$ & glabres & glabres & glabres & glabres & glabres & glabres & glabres & glabres & glabres & glabres & glabres & glabres & $\begin{array}{c}\text { glabres, } \\
\text { mais } \\
\text { souvent } \\
\text { ciliés sous } \\
\text { l'infloresce } \\
\text { nce }\end{array}$ & glabres & glabres & glabres & glabres & glabres & glabres \\
\hline
\end{tabular}


A. NGOM et al. / Int. J. Biol. Chem. Sci. 10(1): 58-86, 2016

Tableau 2 : Caractères morphologiques de la feuille des différentes espèces de Digitaria étudiées.

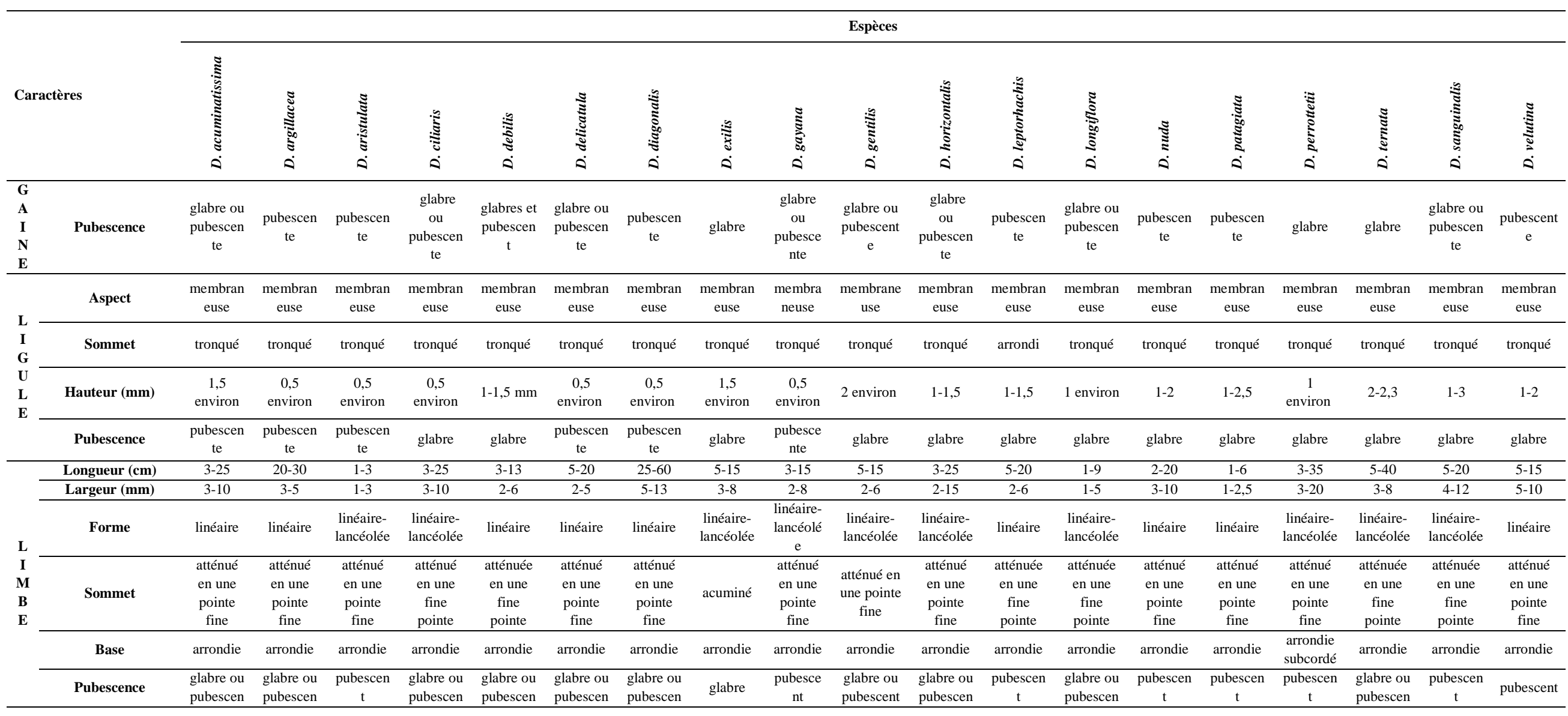


A. NGOM et al. / Int. J. Biol. Chem. Sci. 10(1): 58-86, 2016

\begin{tabular}{|c|c|c|c|c|c|c|c|c|c|c|c|c|c|c|c|c|c|c|c|c|}
\hline & & $\mathrm{t}$ & $\mathrm{t}$ & & $\mathrm{t}$ & $t$ & $\mathrm{t}$ & $\mathrm{t}$ & & & & $\mathrm{t}$ & & $\mathrm{t}$ & & & & $\mathrm{t}$ & & \\
\hline \multirow{3}{*}{ 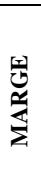 } & Consistance & $\begin{array}{c}\text { cartilagin } \\
\text { euse }\end{array}$ & $\begin{array}{c}\text { cartilagin } \\
\text { euse }\end{array}$ & $\begin{array}{c}\text { cartilagin } \\
\text { euse }\end{array}$ & $\begin{array}{l}\text { cartilagin } \\
\text { euse }\end{array}$ & $\begin{array}{c}\text { cartilagin } \\
\text { euse }\end{array}$ & $\begin{array}{c}\text { cartilagin } \\
\text { euse }\end{array}$ & $\begin{array}{l}\text { cartilagin } \\
\text { euse }\end{array}$ & $\begin{array}{l}\text { cartilagin } \\
\text { euse }\end{array}$ & $\begin{array}{c}\text { cartilagi } \\
\text { neuse }\end{array}$ & $\begin{array}{c}\text { cartilagine } \\
\text { uge }\end{array}$ & $\begin{array}{l}\text { cartilagin } \\
\text { euse }\end{array}$ & $\begin{array}{c}\text { cartilagin } \\
\text { euse }\end{array}$ & $\begin{array}{l}\text { cartilagin } \\
\text { euse }\end{array}$ & $\begin{array}{l}\text { cartilagin } \\
\text { euse }\end{array}$ & $\begin{array}{c}\text { cartilagin } \\
\text { euse }\end{array}$ & $\begin{array}{c}\text { cartilagin } \\
\text { euse }\end{array}$ & $\begin{array}{l}\text { cartilagin } \\
\text { euse }\end{array}$ & $\begin{array}{l}\text { cartilagin } \\
\text { euse }\end{array}$ & $\begin{array}{c}\text { cartilagin } \\
\text { euse }\end{array}$ \\
\hline & Surface & scabre & scabre & scabre & scabre & lisse & scabre & scabre & scabre & scabre & scabre & scabre & scabre & scabre & scabre & scabre & scabre & scabre & scabre & scabre \\
\hline & Pubescence & glabre & glabre & glabre & glabre & glabre & glabre & glabre & glabre & $\begin{array}{c}\text { pubesce } \\
\text { nte }\end{array}$ & glabre & glabre & $\begin{array}{c}\text { pubescen } \\
\text { te }\end{array}$ & glabre & glabre & glabre & glabre & glabre & glabre & glabre \\
\hline
\end{tabular}

Tableau 3 : Caractères morphologiques de l'inflorescence générale des différentes espèces de Digitaria étudiées.

\begin{tabular}{|c|c|c|c|c|c|c|c|c|c|c|c|c|c|c|c|c|c|c|c|c|}
\hline \multirow{2}{*}{\multicolumn{2}{|c|}{ Caractères }} & \multicolumn{19}{|c|}{ Espèces } \\
\hline & & 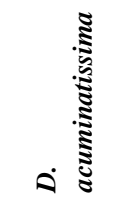 & 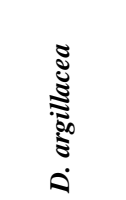 & 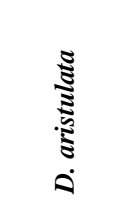 & 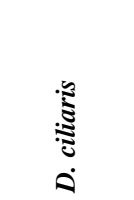 & \begin{tabular}{l}
$\vdots$ \\
\multirow{3}{*}{} \\
0
\end{tabular} & 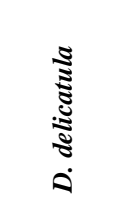 & 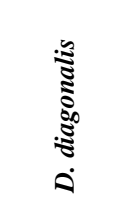 & 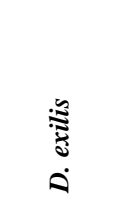 & 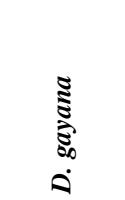 & 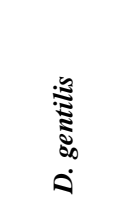 & 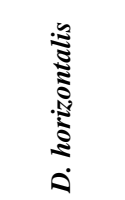 & 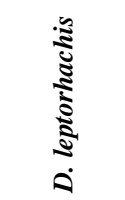 & 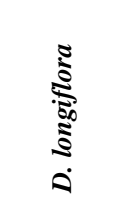 & $\begin{array}{c}\mathbb{\Xi} \\
\mathbb{\Xi} \\
0\end{array}$ & 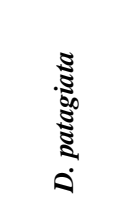 & 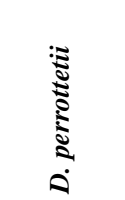 & $\frac{a}{5}$ & 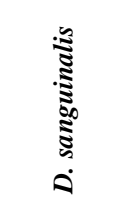 & 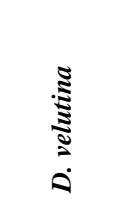 \\
\hline \multicolumn{2}{|c|}{ Type } & racèmes & racèmes & racèmes & racèmes & racèmes & racèmes & racèmes & racèmes & racèmes & racèmes & racèmes & racèmes & racèmes & racèmes & racèmes & racèmes & racèmes & racèmes & racèmes \\
\hline \multicolumn{2}{|c|}{ Nombre } & $2-20$ & $5-12$ & $2-6$ & $5-12$ & $3-20$ & $3-10$ & $10-50$ & $2-5$ & $2-6$ & $6-10$ & $4-15$ & $5-12$ & $2-4$ & $2-20$ & 1 & $\mathrm{n}$ & $2-11$ & $4-12$ & $2-14$ \\
\hline \multicolumn{2}{|c|}{ Organisation } & $\begin{array}{l}\text { digités ou } \\
\text { disposés } \\
\text { le long de } \\
\text { l'axe } \\
\text { principal }\end{array}$ & digités & digités & $\begin{array}{l}\text { digités } \\
\text { ou } \\
\text { subdigit } \\
\text { és }\end{array}$ & $\begin{array}{l}\text { subdigit } \\
\text { és }\end{array}$ & digités & $\begin{array}{l}\text { disposés } \\
\text { le long } \\
\text { de l'axe } \\
\text { principal }\end{array}$ & digités & $\begin{array}{l}\text { digités } \\
\text { ou } \\
\text { subdigit } \\
\text { és }\end{array}$ & $\begin{array}{c}\text { digités } \\
\text { ou } \\
\text { disposés } \\
\text { le long } \\
\text { de l'axe } \\
\text { principal }\end{array}$ & $\begin{array}{l}\text { digités } \\
\text { ou } \\
\text { subdigit } \\
\text { és }\end{array}$ & $\begin{array}{l}\text { subdigit } \\
\text { és }\end{array}$ & digités & digités & $\begin{array}{l}\text { un seul } \\
\text { racème }\end{array}$ & $\begin{array}{c}\text { verticilé } \\
\text { s, } \\
\text { disposés } \\
\text { le long } \\
\text { de l'axe } \\
\text { principal }\end{array}$ & digités & $\begin{array}{l}\text { digités } \\
\text { ou } \\
\text { subdigit } \\
\text { és }\end{array}$ & $\begin{array}{c}\text { digités } \\
\text { ou } \\
\text { subdigité } \\
\text { s }\end{array}$ \\
\hline \multicolumn{2}{|c|}{ Longueur (cm) } & $7-25$ & $10-15$ & $2-5$ & $6-22$ & $3-16$ & $4-12$ & $5-25$ & $3-15$ & $3-18$ & $6-10$ & $3-16$ & $4-11$ & $1-10$ & $4-20$ & $1-6$ & $2-11$ & $3-12$ & $5-18$ & $7-18$ \\
\hline $\begin{array}{l}\mathbf{R} \\
\mathbf{A} \\
\mathbf{C}\end{array}$ & Forme & $\begin{array}{l}\text { triquètre, } \\
\text { ailé }\end{array}$ & $\begin{array}{l}\text { triquètre, } \\
\text { non ailé }\end{array}$ & $\begin{array}{l}\text { triquètre, } \\
\text { ailé }\end{array}$ & $\begin{array}{l}\text { triquètre, } \\
\text { non ailé }\end{array}$ & $\begin{array}{l}\text { triquètre, } \\
\text { non ailé }\end{array}$ & $\begin{array}{l}\text { triquètre, } \\
\text { non ailé }\end{array}$ & $\begin{array}{l}\text { triquètre, } \\
\text { non ailé }\end{array}$ & $\begin{array}{l}\text { triquètre, } \\
\text { non ailé }\end{array}$ & $\begin{array}{l}\text { triquètre, } \\
\text { ailé }\end{array}$ & $\begin{array}{l}\text { triquètre, } \\
\text { ailé }\end{array}$ & $\begin{array}{l}\text { triquètre, } \\
\text { non ailé }\end{array}$ & $\begin{array}{l}\text { triquètre, } \\
\text { non ailé }\end{array}$ & $\begin{array}{l}\text { triquètre, } \\
\text { non ailé }\end{array}$ & $\begin{array}{l}\text { triquètre, } \\
\text { ailé }\end{array}$ & $\begin{array}{l}\text { triquètre, } \\
\text { non ailé }\end{array}$ & $\begin{array}{l}\text { triquètre, } \\
\text { non ailé }\end{array}$ & $\begin{array}{l}\text { triquètre } \\
\text { ailé }\end{array}$ & $\begin{array}{l}\text { triquètre, } \\
\text { ailé }\end{array}$ & $\begin{array}{l}\text { triquètre, } \\
\text { ailé }\end{array}$ \\
\hline $\begin{array}{l}\text { I } \\
\text { S }\end{array}$ & Marge & scabre & scabre & scabre & scabre & scabre & scabre & scabre & scabre & scabre & scabre & scabre & scabre & scabre & scabre & scabre & scabre & scabre & scabre & scabre \\
\hline
\end{tabular}


A. NGOM et al. / Int. J. Biol. Chem. Sci. 10(1): 58-86, 2016

Tableau 4 : Caractères morphologiques de l'épillet des différentes espèces de Digitaria étudiées.

\begin{tabular}{|c|c|c|c|c|c|c|c|c|c|c|c|c|c|c|c|c|c|c|c|}
\hline \multirow[b]{2}{*}{ Caractères } & \multicolumn{19}{|c|}{ Espèces } \\
\hline & 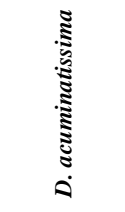 & 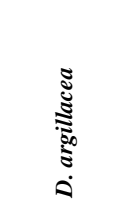 & 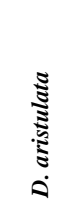 & 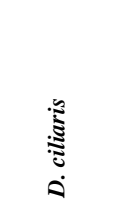 & 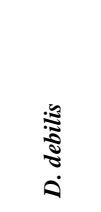 & 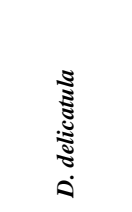 & 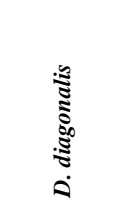 & 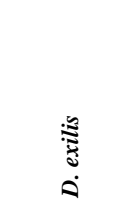 & 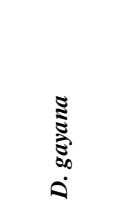 & 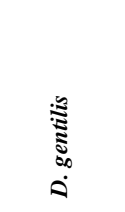 & $\frac{0}{3}$ & $\frac{3}{3}$ & 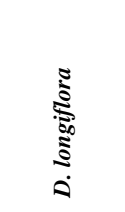 & $\underset{\Xi}{\Xi}$ & 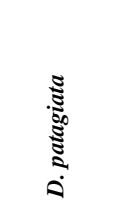 & 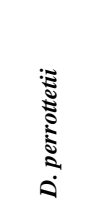 & $\frac{\Xi}{5}$ & 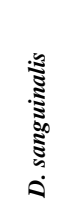 & 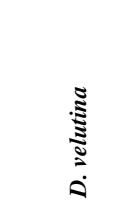 \\
\hline Longueur (mm) & $2,5-4,5$ & $1,8-2$ & $1,5-2$ & $2,7-3,4$ & $2-4,5$ & $1,8-2$ & $1,4-2$ & $1,5-2$ & $2-3$ & $3-4$ & $2-2,5$ & $1,4-2,2$ & $1,2-1,8$ & 2 à 2,8 & $2-3$ & $1,8-2,3$ & $1,8-2,7$ & $\begin{array}{c}3- \\
3,5\end{array}$ & $1,5-2,1$ \\
\hline Forme & $\begin{array}{l}\text { lancéolée- } \\
\text { elliptique }\end{array}$ & elliptique & $\begin{array}{l}\text { lancé } \\
\text { olée }\end{array}$ & elliptique & $\begin{array}{c}\text { lancéolé } \\
\mathrm{e}\end{array}$ & $\begin{array}{l}\text { lancéolée- } \\
\text { elliptique }\end{array}$ & $\begin{array}{l}\text { elliptique- } \\
\text { oblongue }\end{array}$ & $\begin{array}{l}\text { elliptique- } \\
\text { oblongue }\end{array}$ & ovale & lancéolée & lancéolée & elliptique & $\begin{array}{l}\text { ovale- } \\
\text { elliptique }\end{array}$ & elliptique & $\begin{array}{c}\text { lancéolé } \\
\text { e- } \\
\text { oblongu } \\
\text { e }\end{array}$ & $\begin{array}{l}\text { elliptiq } \\
\text { ue- } \\
\text { oblong } \\
\text { ue }\end{array}$ & $\begin{array}{l}\text { ovale- } \\
\text { elliptique }\end{array}$ & $\begin{array}{c}\text { lanc } \\
\text { éolé } \\
\text { e- } \\
\text { elli } \\
\text { ptiq } \\
\text { ue }\end{array}$ & $\begin{array}{l}\text { oblongue- } \\
\text { lanceolée }\end{array}$ \\
\hline Sommet & acuminé & aigu & $\begin{array}{l}\text { acumi } \\
\text { né }\end{array}$ & $\begin{array}{c}\text { aigus- } \\
\text { acuminés }\end{array}$ & acuminé & $\begin{array}{l}\text { aigu (enflé } \\
\text { en massue } \\
\text { et parfois } \\
\text { mucroné) }\end{array}$ & aigu & subacuminé & $\begin{array}{l}\text { subacumi } \\
\text { né }\end{array}$ & aigu & aigu & aigu & aigu & aigu & aigu & aigu & $\begin{array}{l}\text { obtus ou } \\
\text { subobtus }\end{array}$ & $\begin{array}{c}\text { aig } \\
\mathrm{u}\end{array}$ & subaigu \\
\hline Pubescence & pubescents & pubescents & $\begin{array}{l}\text { pubes } \\
\text { cents }\end{array}$ & $\begin{array}{c}\text { pubescen } \\
\text { ts }\end{array}$ & $\begin{array}{c}\text { pubesce } \\
\text { nts }\end{array}$ & pubescents & glabres & glabres & $\begin{array}{c}\text { pubescent } \\
\mathrm{s}\end{array}$ & $\begin{array}{c}\text { pubescent } \\
\mathrm{s}\end{array}$ & $\begin{array}{c}\text { pubescent } \\
\mathrm{s}\end{array}$ & $\begin{array}{c}\text { pubescent } \\
\mathrm{s}\end{array}$ & $\begin{array}{c}\text { pubescent } \\
\mathrm{s}\end{array}$ & pubescents & $\begin{array}{c}\text { pubesce } \\
\text { nts }\end{array}$ & $\begin{array}{c}\text { pubesc } \\
\text { ents }\end{array}$ & $\begin{array}{c}\text { pubescent } \\
\mathrm{s}\end{array}$ & $\begin{array}{l}\text { pub } \\
\text { esc } \\
\text { ents }\end{array}$ & pubescents \\
\hline
\end{tabular}


A. NGOM et al. / Int. J. Biol. Chem. Sci. 10(1): 58-86, 2016

\begin{tabular}{|c|c|c|c|c|c|c|c|c|c|c|c|c|c|c|c|c|c|c|c|c|}
\hline Nate & e des poils & $\begin{array}{c}\text { poils } \\
\text { apprimés }\end{array}$ & $\begin{array}{l}\text { poils soyeux } \\
\text { rigides }\end{array}$ & $\begin{array}{l}\text { poils } \\
\text { courts } \\
\text { verru } \\
\text { queux }\end{array}$ & $\begin{array}{c}\text { poils } \\
\text { apprimés }\end{array}$ & $\begin{array}{l}\text { poils } \\
\text { apprimé } \\
\text { s }\end{array}$ & $\begin{array}{c}\text { poils } \\
\text { soyeux } \\
\text { souples à } \\
\text { sommet } \\
\text { enflé en } \\
\text { massue }\end{array}$ & absents & absents & $\begin{array}{c}\text { poils } \\
\text { longs et } \\
\text { soyeux } \\
\text { argentés } \\
\text { ou rouges }\end{array}$ & $\begin{array}{c}\text { poils } \\
\text { apprimés }\end{array}$ & $\begin{array}{c}\text { poils } \\
\text { apprimés }\end{array}$ & $\begin{array}{l}\text { poils } \\
\text { soyeux- } \\
\text { argentés }\end{array}$ & $\begin{array}{c}\text { poils } \\
\text { courts } \\
\text { verruqueu } \\
\text { x }\end{array}$ & $\begin{array}{c}\text { poils } \\
\text { apprimés à } \\
\text { marges } \\
\text { ciliées- } \\
\text { soyeuses }\end{array}$ & $\begin{array}{c}\text { poils } \\
\text { courts } \\
\text { verruqu } \\
\text { eux }\end{array}$ & $\begin{array}{l}\text { poils } \\
\text { apprim } \\
\text { és }\end{array}$ & $\begin{array}{c}\text { poils } \\
\text { soyeux } \\
\text { souples à } \\
\text { sommet } \\
\text { enflé en } \\
\text { massue }\end{array}$ & $\begin{array}{l}\text { poil } \\
\mathrm{s} \\
\text { app } \\
\text { rim } \\
\text { és }\end{array}$ & poils apprimés \\
\hline $\begin{array}{l}\mathbf{P} \\
\mathbf{E} \\
\mathbf{D}\end{array}$ & $\begin{array}{l}\text { Dimension } \\
\mathrm{s}\end{array}$ & inégaux & inégaux & $\begin{array}{l}\text { inéga } \\
\text { ux }\end{array}$ & inégaux & inégaux & inégaux & inégaux & inégaux & inégaux & inégaux & inégaux & inégaux & inégaux & inégaux & inégaux & $\begin{array}{l}\text { inégau } \\
\quad \mathrm{x}\end{array}$ & inégaux & $\begin{array}{c}\text { iné } \\
\text { gau } \\
\mathrm{x}\end{array}$ & inégaux \\
\hline I & Surface & scabre & scabre & lisse & scabre & scabre & scabre & scabre & lisse & scabre & scabre & scabre & scabre & lisse & scabre & scabre & scabre & scabre & $\begin{array}{l}\text { sca } \\
\text { bre }\end{array}$ & scabre \\
\hline $\begin{array}{l}\text { C } \\
\text { E } \\
\text { L }\end{array}$ & $\begin{array}{l}\text { Pubescenc } \\
\text { e }\end{array}$ & glabres & glabres & $\begin{array}{c}\text { glabre } \\
\mathrm{s}\end{array}$ & glabres & glabres & glabres & pubescents & $\begin{array}{l}\text { glabres ou } \\
\text { pubescents }\end{array}$ & glabres & glabres & glabres & glabres & glabres & $\begin{array}{l}\text { glabres ou } \\
\text { pubescents }\end{array}$ & glabres & glabres & $\begin{array}{c}\text { pubescent } \\
\mathrm{s}\end{array}$ & $\begin{array}{c}\text { gla } \\
\text { bres }\end{array}$ & glabres \\
\hline $\begin{array}{l}\text { L } \\
\text { E } \\
\text { S }\end{array}$ & $\begin{array}{l}\text { Longueur } \\
\text { des poils }\end{array}$ & absents & absents & $\begin{array}{c}\text { absent } \\
\text { s }\end{array}$ & absents & absents & absents & $\begin{array}{l}\text { aussi longs ou } \\
\text { dépassant le } \\
\text { sommet de } \\
\text { l'épillet }\end{array}$ & $\begin{array}{l}\text { absent ou } \\
\text { plus courts } \\
\text { que l'épillet }\end{array}$ & absents & absents & absents & absents & absents & $\begin{array}{l}\text { absents ou } \\
\text { plus courts } \\
\text { que l'épillet }\end{array}$ & absents & $\begin{array}{c}\text { absent } \\
\text { s }\end{array}$ & $\begin{array}{c}\text { plus } \\
\text { courts que } \\
\text { l'épillet }\end{array}$ & $\begin{array}{l}\text { abs } \\
\text { ents }\end{array}$ & absents \\
\hline
\end{tabular}


A. NGOM et al. / Int. J. Biol. Chem. Sci. 10(1): 58-86, 2016

Tableau 5 : Caractères morphologiques des glumes des différentes espèces de Digitaria étudiées.

\begin{tabular}{|c|c|c|c|c|c|c|c|c|c|c|c|c|c|c|c|c|c|c|c|c|}
\hline \multirow{2}{*}{\multicolumn{2}{|c|}{ Caractères }} & \multicolumn{19}{|c|}{ Espèces } \\
\hline & & 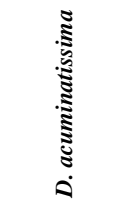 & 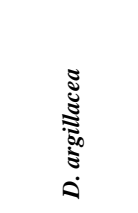 & 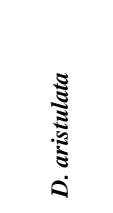 & 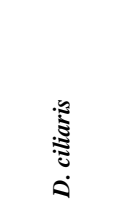 & 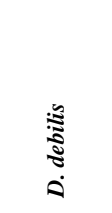 & 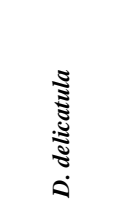 & 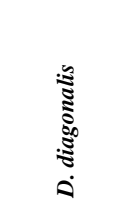 & 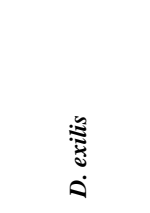 & 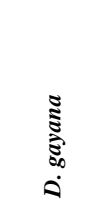 & 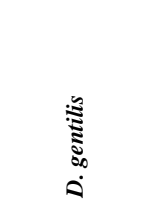 & 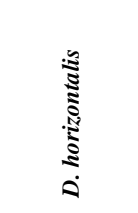 & 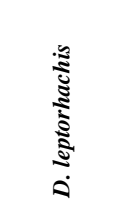 & 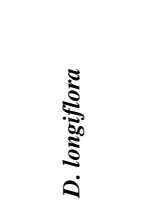 & $\underset{\Xi}{\Xi}$ & 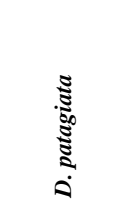 & 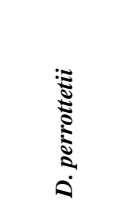 & $\frac{5}{5}$ & 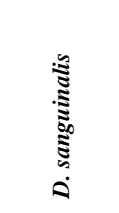 & 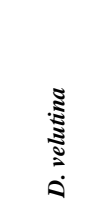 \\
\hline \multirow{3}{*}{ 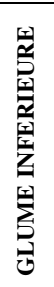 } & Forme & écaille & \multirow{3}{*}{ absente } & $\begin{array}{c}\text { absente } \\
\text { ou en } \\
\text { bourrelet }\end{array}$ & ovale & $\begin{array}{l}\text { absente } \\
\text { ou en } \\
\text { écaille }\end{array}$ & \multirow{3}{*}{ absente } & \multirow{3}{*}{ absente } & bourrelet & $\begin{array}{c}\text { bourrel } \\
\text { et }\end{array}$ & $\begin{array}{l}\text { absente ou } \\
\text { en bourrelet }\end{array}$ & $\begin{array}{c}\text { absente ou } \\
\text { en } \\
\text { bourrelet }\end{array}$ & anneau & $\begin{array}{l}\text { absente ou } \\
\text { en une } \\
\text { minuscule } \\
\text { membrane }\end{array}$ & $\begin{array}{c}\text { absente } \\
\text { ou en } \\
\text { bourrelet }\end{array}$ & écaille & \multirow{3}{*}{ absente } & \multirow{3}{*}{ absente } & $\begin{array}{l}\text { absente } \\
\text { ou en } \\
\text { bourrelet }\end{array}$ & $\begin{array}{c}\text { absente } \\
\text { ou en } \\
\text { bourrelet }\end{array}$ \\
\hline & Sommet & ovale & & tronquée & aigue & tronquée & & & tronquée & $\begin{array}{c}\text { tronqué } \\
\mathrm{e}\end{array}$ & tronquée & tronquée & tronquée & tronquée & tronquée & tronquée & & & tronquée & tronquée \\
\hline & Nervures & $\begin{array}{c}\text { sans } \\
\text { nervure }\end{array}$ & & $\begin{array}{c}\text { sans } \\
\text { nervure }\end{array}$ & $\begin{array}{c}\text { sans } \\
\text { nervure }\end{array}$ & $\begin{array}{c}\text { sans } \\
\text { nervure }\end{array}$ & & & sans nervure & $\begin{array}{c}\text { sans } \\
\text { nervure }\end{array}$ & $\begin{array}{c}\text { sans } \\
\text { nervure }\end{array}$ & $\begin{array}{c}\text { sans } \\
\text { nervure }\end{array}$ & $\begin{array}{c}\text { sans } \\
\text { nervure }\end{array}$ & $\begin{array}{c}\text { sans } \\
\text { nervure }\end{array}$ & $\begin{array}{c}\text { sans } \\
\text { nervure }\end{array}$ & $\begin{array}{c}\text { sans } \\
\text { nervure }\end{array}$ & & & $\begin{array}{c}\text { sans } \\
\text { nervure }\end{array}$ & $\begin{array}{c}\text { sans } \\
\text { nervure }\end{array}$ \\
\hline \multirow{5}{*}{ 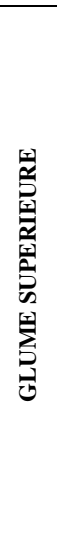 } & Forme & $\begin{array}{c}\text { lancéolée- } \\
\text { ovale }\end{array}$ & lancéolée & $\begin{array}{c}\text { oblongu } \\
\mathrm{e}\end{array}$ & $\begin{array}{l}\text { linéaire- } \\
\text { lancéolée }\end{array}$ & $\begin{array}{c}\text { lancéolé } \\
\mathrm{e}\end{array}$ & $\begin{array}{c}\text { ovale- } \\
\text { oblongue }\end{array}$ & ovale & oblongue & $\begin{array}{c}\text { oblong } \\
\text { ue- } \\
\text { lancéol } \\
\text { ée } \\
\end{array}$ & elliptique & $\begin{array}{l}\text { linéaire- } \\
\text { lancéolée }\end{array}$ & elliptique & elliptique & $\begin{array}{l}\text { oblongue- } \\
\text { triangulai } \\
\text { re }\end{array}$ & ovale & elliptique & $\begin{array}{c}\text { oblongue } \\
- \\
\text { lancéolée }\end{array}$ & $\begin{array}{c}\text { lancéolé } \\
\mathrm{e}\end{array}$ & $\begin{array}{c}\text { lancéolé } \\
\text { e }\end{array}$ \\
\hline & Sommet & acuminé & aigu & $\begin{array}{c}\text { obtus- } \\
\text { subaigu }\end{array}$ & aigu & acuminé & aigu & obtus & obtus & aigu & aigu & aigu & obtus & aigu & aigu & aigu & aigu & obtus & obtus & aigu \\
\hline & $\begin{array}{c}\text { Longueur } \\
\text { relative }\end{array}$ & $\begin{array}{c}\text { 1/3-1/2 de } \\
\text { la } \\
\text { longueur } \\
\text { de } \\
\text { l'épillet }\end{array}$ & $\begin{array}{l}\text { 3/4 de la } \\
\text { longueur de } \\
\text { l'épillet }\end{array}$ & $\begin{array}{c}1 / 3 \text { de la } \\
\text { longueur } \\
\text { de } \\
\text { l'épillet }\end{array}$ & $\begin{array}{c}1 / 2-3 / 4 \mathrm{de} \\
\text { la } \\
\text { longueur } \\
\text { de } \\
\text { l'épillet }\end{array}$ & $\begin{array}{c}\text { de la } \\
\text { longueur } \\
\text { de } \\
\text { l'épillet }\end{array}$ & $\begin{array}{c}\text { 3/4 de la } \\
\text { longueur } \\
\text { de } \\
\text { l'épillet }\end{array}$ & $\begin{array}{c}\text { 1/4-1/3 } \\
\text { de la } \\
\text { longueur } \\
\text { de l'épillet }\end{array}$ & $\begin{array}{l}\text { de la } \\
\text { longueur de } \\
\text { l'épillet }\end{array}$ & $\begin{array}{c}4 / 5 \mathrm{de} \\
\text { la } \\
\text { longue } \\
\text { ur de } \\
\text { l'épille } \\
\text { t }\end{array}$ & $\begin{array}{c}\text { de la } \\
\text { longueur de } \\
\text { l'épillet }\end{array}$ & $\begin{array}{l}\text { 1/2 de la } \\
\text { longueur } \\
\text { de l'épillet }\end{array}$ & $\begin{array}{c}\text { de la } \\
\text { longueur } \\
\text { de } \\
\text { l'épillet }\end{array}$ & $\begin{array}{c}\text { de la } \\
\text { longueur de } \\
\text { l'épillet }\end{array}$ & $\begin{array}{l}1 / 2 \text { ou } 3 / 4 \\
\text { de la } \\
\text { longueur } \\
\text { de } \\
\text { l'épillet }\end{array}$ & $\begin{array}{c}\text { de même } \\
\text { longueur } \\
\text { que } \\
\text { l'épillet }\end{array}$ & $\begin{array}{l}\text { 3/4-9/10 } \\
\text { de la } \\
\text { longueur } \\
\text { de } \\
\text { l'épillet }\end{array}$ & $\begin{array}{c}\text { 2/3-4/5 } \\
\text { de la } \\
\text { longueur } \\
\quad \text { de } \\
\text { l'épillet }\end{array}$ & $\begin{array}{l}\text { 1/3-1/2 } \\
\text { de la } \\
\text { longueur } \\
\quad \text { de } \\
\text { l'épillet }\end{array}$ & $\begin{array}{c}\text { 2/3-3/4 } \\
\text { de la } \\
\text { longueur } \\
\text { de } \\
\text { l'épillet }\end{array}$ \\
\hline & Nervures & $\begin{array}{c}\text { 3-5- } \\
\text { nervée }\end{array}$ & 3-nervée & 3-nervée & 3-nervée & $\begin{array}{c}5- \\
7 \text { nervée }\end{array}$ & 3-nervée & $\begin{array}{l}\text { dépourvu } \\
\text { e de } \\
\text { nervure }\end{array}$ & $\begin{array}{c}\text { 3-5 nervée } \\
\text { (rarement 3) }\end{array}$ & $\begin{array}{c}\text { 3- } \\
\text { nervée }\end{array}$ & 3-nervée & 3-nervée & 5-nervée & 5-nervée & 3-nervée & 5-nervée & 3-nervée & 3-nervée & 3-nervée & 3-nervée \\
\hline & $\begin{array}{l}\text { PUBESCE } \\
\text { NCE }\end{array}$ & $\begin{array}{c}\text { pubescent } \\
\mathrm{e}\end{array}$ & pubescente & $\begin{array}{c}\text { pubesce } \\
\text { nte }\end{array}$ & $\begin{array}{c}\text { pubescent } \\
\mathrm{e}\end{array}$ & $\begin{array}{l}\text { pubesce } \\
\text { nte }\end{array}$ & $\begin{array}{l}\text { pubescen } \\
\text { te }\end{array}$ & glabre & glabre & $\begin{array}{c}\text { pubesc } \\
\text { ente }\end{array}$ & pubescente & $\begin{array}{c}\text { pubescent } \\
\mathrm{e}\end{array}$ & $\begin{array}{c}\text { pubescent } \\
\mathrm{e}\end{array}$ & pubescente & $\begin{array}{c}\text { pubescent } \\
\mathrm{e}\end{array}$ & $\begin{array}{c}\text { pubescent } \\
\mathrm{e}\end{array}$ & $\begin{array}{c}\text { pubescent } \\
\mathrm{e}\end{array}$ & $\begin{array}{c}\text { pubescen } \\
\text { te }\end{array}$ & $\begin{array}{c}\text { pubesce } \\
\text { nte }\end{array}$ & $\begin{array}{c}\text { pubesce } \\
\text { nte }\end{array}$ \\
\hline
\end{tabular}


Tableau 6 : Caractères morphologiques des fleurs des différentes espèces de Digitaria étudiées.

\begin{tabular}{|c|c|c|c|c|c|c|c|c|c|c|c|c|c|c|c|c|c|c|c|c|c|}
\hline & & & & & & & & & & & Esp & èces & & & & & & & & & \\
\hline & & Iractères & D. & $\begin{array}{c}D . \\
\text { aroilla }\end{array}$ & $\begin{array}{c}D . \\
\text { aristulg }\end{array}$ & $D$ & $D$ & & & $D$. & & $D$ & $D$. & $\begin{array}{c}D . \\
\text { lentorha }\end{array}$ & $\begin{array}{c}D . \\
\text { Dopiflo }\end{array}$ & $D$. & $\begin{array}{c}D . \\
\text { nataoi }\end{array}$ & $\begin{array}{c}D . \\
\text { nerratt }\end{array}$ & $D$. & $\begin{array}{c}D . \\
\text { sanoui }\end{array}$ & $D$. \\
\hline & & Fertilité & stérile & stérile & stérile & stérile & stérile & stérile & stérile & stérile & stérile & stérile & stérile & stérile & stérile & stérile & stérile & stérile & stérile & stérile & stérile \\
\hline & & Forme & elliptique & $\begin{array}{l}\text { ellipti } \\
\text { que }\end{array}$ & $\begin{array}{c}\text { oblong } \\
\text { ue- } \\
\text { lancéol } \\
\text { ée }\end{array}$ & $\begin{array}{l}\text { elliptiqu } \\
\text { e }\end{array}$ & $\begin{array}{l}\text { elliptiqu } \\
\text { e }\end{array}$ & $\begin{array}{c}\text { lancéolée } \\
- \\
\text { elliptique }\end{array}$ & $\begin{array}{c}\text { lancéolée- } \\
\text { ovale }\end{array}$ & $\begin{array}{l}\text { ellipti } \\
\text { que }\end{array}$ & $\begin{array}{l}\text { elliptiqu } \\
\mathrm{e}\end{array}$ & $\begin{array}{l}\text { elliptiq } \\
\text { ue }\end{array}$ & $\begin{array}{l}\text { ellipti } \\
\text { que }\end{array}$ & $\begin{array}{l}\text { elliptiqu } \\
\text { e }\end{array}$ & $\begin{array}{l}\text { elliptiq } \\
\text { ue }\end{array}$ & $\begin{array}{c}\text { oblong } \\
\text { ue- } \\
\text { lancéol } \\
\text { ée }\end{array}$ & $\begin{array}{l}\text { ellipti } \\
\text { que }\end{array}$ & $\begin{array}{l}\text { elliptiq } \\
\text { ue }\end{array}$ & $\begin{array}{c}\text { oblongu } \\
\text { e- } \\
\text { elliptiq } \\
\text { ue }\end{array}$ & $\begin{array}{c}\text { ovale- } \\
\text { oblong } \\
\text { ue }\end{array}$ & oblongue \\
\hline 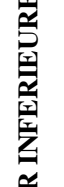 & 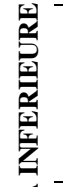 & $\begin{array}{l}\text { Longueur } \\
\text { relative }\end{array}$ & $\begin{array}{c}\text { aussi } \\
\text { longue que } \\
\text { l'épillet }\end{array}$ & $\begin{array}{c}\text { aussi } \\
\text { longue } \\
\text { que } \\
\text { l'épill } \\
\text { et }\end{array}$ & $\begin{array}{c}4 / 5 \mathrm{de} \\
\text { la } \\
\text { longue } \\
\text { ur de } \\
\text { l'épillet }\end{array}$ & $\begin{array}{l}\text { aussi } \\
\text { longue } \\
\text { que } \\
\text { l'épillet }\end{array}$ & $\begin{array}{c}7 / 10 \text { à } \\
4 / 5 \text { de la } \\
\text { longueu } \\
\text { r de } \\
\text { l'épillet }\end{array}$ & $\begin{array}{c}\text { aussi } \\
\text { longue } \\
\text { que } \\
\text { l'épillet }\end{array}$ & $\begin{array}{l}4 / 5 \text { à } 1 \text { fois } \\
\text { la longueur } \\
\text { de l'épillet }\end{array}$ & $\begin{array}{c}\text { aussi } \\
\text { longu } \\
\text { e que } \\
\text { l'épill } \\
\text { et }\end{array}$ & $\begin{array}{l}\text { aussi } \\
\text { longue } \\
\text { que } \\
\text { l'épillet }\end{array}$ & $\begin{array}{l}\text { aussi } \\
\text { longue } \\
\text { que } \\
\text { l'épillet }\end{array}$ & $\begin{array}{l}\text { aussi } \\
\text { longue } \\
\text { que } \\
\text { l'épill } \\
\text { et }\end{array}$ & $\begin{array}{c}\text { aussi } \\
\text { longue } \\
\text { que } \\
\text { l'épillet }\end{array}$ & $\begin{array}{l}\text { aussi } \\
\text { longue } \\
\text { que } \\
\text { l'épillet }\end{array}$ & $\begin{array}{c}\text { aussi } \\
\text { longue } \\
\text { que } \\
\text { l'épillet }\end{array}$ & $\begin{array}{c}\text { aussi } \\
\text { longu } \\
\text { e que } \\
\text { l'épill } \\
\text { et }\end{array}$ & $\begin{array}{c}\text { aussi } \\
\text { longue } \\
\text { que } \\
\text { l'épille } \\
\text { t }\end{array}$ & $\begin{array}{l}\text { aussi } \\
\text { longue } \\
\text { que } \\
\text { l'épillet }\end{array}$ & $\begin{array}{c}\text { aussi } \\
\text { longue } \\
\text { que } \\
\text { l'épille } \\
\text { t }\end{array}$ & $\begin{array}{l}\text { aussi } \\
\text { longue } \\
\text { que } \\
\text { l'épillet }\end{array}$ \\
\hline 空 & 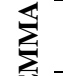 & Sommet & aigu & aigu & aigu & aigu & aigu & aigu & aigu & aigu & aigu & aigu & aigu & aigu & aigu & aigu & aigu & aigu & $\begin{array}{c}\text { subobtu } \\
\mathrm{s}\end{array}$ & aigu & aigu \\
\hline & & Nervures & 7-nervée & $\begin{array}{c}\text { 7- } \\
\text { nervée }\end{array}$ & $\begin{array}{c}5-7- \\
\text { nervée }\end{array}$ & 7-nervée & 7-nervée & 5-nervée & $\begin{array}{l}\text { 3-5-7- } \\
\text { nervée }\end{array}$ & $\begin{array}{c}7-9 \\
\text { nervé } \\
\text { e }\end{array}$ & $\begin{array}{c}5-7 \\
\text { nervée }\end{array}$ & $\begin{array}{c}5- \\
\text { nervée }\end{array}$ & $\begin{array}{c}\text { 7- } \\
\text { nervée }\end{array}$ & 7-nervée & $\begin{array}{c}\text { 7- } \\
\text { nervée }\end{array}$ & $\begin{array}{c}5-7- \\
\text { nervée }\end{array}$ & $\begin{array}{c}5- \\
\text { nervé } \\
\text { e }\end{array}$ & $\begin{array}{c}5-7- \\
\text { nervée }\end{array}$ & $\begin{array}{c}\text { 5-9- } \\
\text { nervée }\end{array}$ & $\begin{array}{c}\text { 7- } \\
\text { nervée }\end{array}$ & 7-nervée \\
\hline & & Pubescence & $\begin{array}{c}\text { pubescent } \\
\mathrm{e}\end{array}$ & $\begin{array}{l}\text { pubes } \\
\text { cente }\end{array}$ & $\begin{array}{c}\text { pubesc } \\
\text { ente }\end{array}$ & $\begin{array}{c}\text { pubesce } \\
\text { nte }\end{array}$ & $\begin{array}{c}\text { pubesce } \\
\text { nte }\end{array}$ & $\begin{array}{c}\text { pubescent } \\
\mathrm{e}\end{array}$ & glabre & glabre & $\begin{array}{c}\text { pubesce } \\
\text { nte }\end{array}$ & $\begin{array}{c}\text { pubesce } \\
\text { nte }\end{array}$ & $\begin{array}{l}\text { pubes } \\
\text { cente }\end{array}$ & $\begin{array}{c}\text { pubesce } \\
\text { nte }\end{array}$ & $\begin{array}{c}\text { pubesce } \\
\text { nte }\end{array}$ & $\begin{array}{c}\text { pubesce } \\
\text { nte }\end{array}$ & $\begin{array}{l}\text { pubes } \\
\text { cente }\end{array}$ & $\begin{array}{c}\text { pubesc } \\
\text { ente }\end{array}$ & $\begin{array}{c}\text { pubesce } \\
\text { nte }\end{array}$ & $\begin{array}{c}\text { pubesc } \\
\text { ente }\end{array}$ & $\begin{array}{c}\text { pubescent } \\
\mathrm{e}\end{array}$ \\
\hline $\begin{array}{l}\mathbf{F} \\
\mathbf{L} \\
\mathbf{E} \\
\mathbf{U}\end{array}$ & $\begin{array}{l}\mathbf{L} \\
\mathbf{E} \\
\mathbf{M} \\
\mathbf{M}\end{array}$ & Forme & ovale & ovale & $\begin{array}{c}\text { oblong } \\
\text { ue- } \\
\text { lancéol } \\
\text { ée }\end{array}$ & 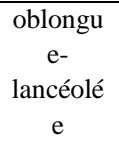 & $\begin{array}{l}\text { elliptiqu } \\
\mathrm{e}\end{array}$ & ovale & ovale & $\begin{array}{l}\text { obova } \\
\text { le }\end{array}$ & $\begin{array}{l}\text { elliptiqu } \\
\mathrm{e}\end{array}$ & $\begin{array}{l}\text { elliptiq } \\
\text { ue }\end{array}$ & $\begin{array}{l}\text { lancéo } \\
\text { lée }\end{array}$ & $\begin{array}{l}\text { elliptiqu } \\
\mathrm{e}\end{array}$ & $\begin{array}{l}\text { elliptiq } \\
\text { ue }\end{array}$ & $\begin{array}{c}\text { oblong } \\
\text { ue- } \\
\text { lancéol } \\
\text { ée }\end{array}$ & $\begin{array}{l}\text { ellipti } \\
\text { que }\end{array}$ & $\begin{array}{c}\text { oboval } \\
\mathrm{e}\end{array}$ & $\begin{array}{l}\text { elliptiq } \\
\text { ue }\end{array}$ & $\begin{array}{c}\text { lancéol } \\
\text { ée }\end{array}$ & elliptique \\
\hline $\begin{array}{l}\mathbf{R} \\
\mathbf{S} \\
\mathbf{U}\end{array}$ & $\begin{array}{l}\mathbf{A} \\
\mathbf{S} \\
\mathbf{U}\end{array}$ & Sommet & acuminé & aigu & $\begin{array}{l}\text { acumin } \\
\text { é }\end{array}$ & aigu & acuminé & $\begin{array}{l}\text { aigu- } \\
\text { mucroné }\end{array}$ & obtus & $\begin{array}{l}\text { obtus- } \\
\text { arron } \\
\text { di }\end{array}$ & $\begin{array}{c}\text { acuminé } \\
\text {-rosté }\end{array}$ & aigu & aigu & aigu & $\begin{array}{l}\text { acumin } \\
\text { é }\end{array}$ & $\begin{array}{c}\text { aigu- } \\
\text { acumin } \\
\text { é }\end{array}$ & aigu & $\begin{array}{l}\text { obtus- } \\
\text { mucron } \\
\text { é }\end{array}$ & aigu & $\begin{array}{l}\text { acumin } \\
\text { é }\end{array}$ & aigu \\
\hline $\begin{array}{l}\mathbf{P} \\
\mathbf{E}\end{array}$ & $\begin{array}{l}\mathbf{P} \\
\mathbf{E}\end{array}$ & $\begin{array}{c}\text { Longueur } \\
\text { relative }\end{array}$ & $\begin{array}{l}\text { inférieure } \\
\text { à la }\end{array}$ & $\begin{array}{c}\text { aussi } \\
\text { longue }\end{array}$ & $\begin{array}{c}\text { inférieu } \\
\text { re à la }\end{array}$ & $\begin{array}{c}\text { aussi } \\
\text { longue }\end{array}$ & $\begin{array}{c}\text { aussi } \\
\text { longue }\end{array}$ & $\begin{array}{c}\text { inférieure } \\
\text { à la }\end{array}$ & $\begin{array}{c}\text { aussi } \\
\text { longue que }\end{array}$ & $\begin{array}{l}\text { aussi } \\
\text { longu }\end{array}$ & $\begin{array}{c}\text { aussi } \\
\text { longue }\end{array}$ & $\begin{array}{l}\text { inférieu } \\
\text { re à la }\end{array}$ & $\begin{array}{l}\text { aussi } \\
\text { longue }\end{array}$ & $\begin{array}{c}\text { aussi } \\
\text { longue }\end{array}$ & $\begin{array}{c}\text { aussi } \\
\text { longue }\end{array}$ & $\begin{array}{c}\text { inférieu } \\
\text { re à la }\end{array}$ & $\begin{array}{c}\text { inférie } \\
\text { ure à }\end{array}$ & $\begin{array}{l}\text { inférie } \\
\text { ure à la }\end{array}$ & $\begin{array}{l}\text { inférieu } \\
\text { re à la }\end{array}$ & $\begin{array}{l}\text { inférie } \\
\text { ure ou }\end{array}$ & $\begin{array}{l}\text { inférieure } \\
\text { ou égale à }\end{array}$ \\
\hline
\end{tabular}


A. NGOM et al. / Int. J. Biol. Chem. Sci. 10(1): 58-86, 2016

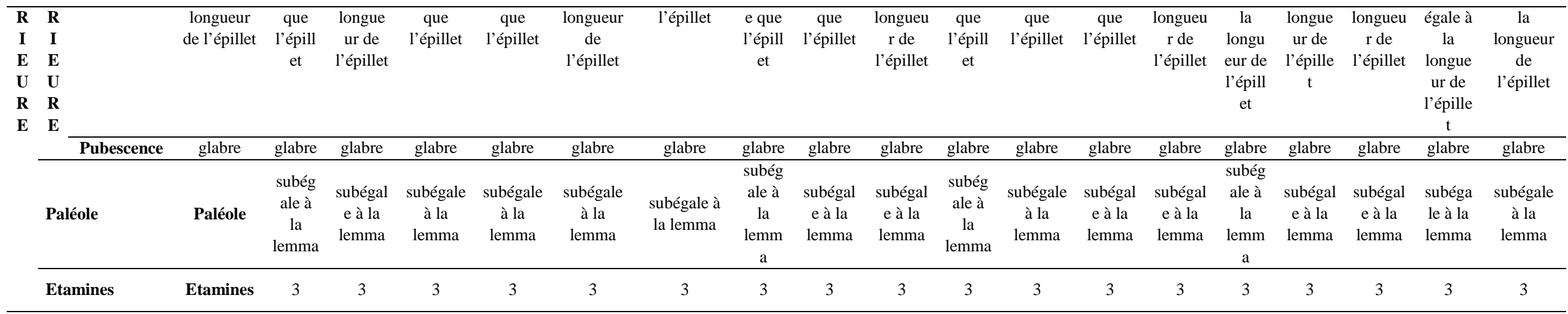


Tableau 7 : Caractères morphologiques du fruit des différentes espèces de Digitaria étudiées.

\begin{tabular}{|c|c|c|c|c|c|c|c|c|c|c|c|c|c|c|c|c|c|c|c|}
\hline \multirow[b]{2}{*}{ Caractères } & \multicolumn{19}{|c|}{ Espèces } \\
\hline & 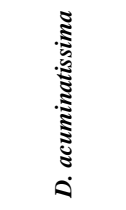 & 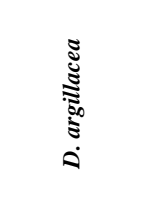 & 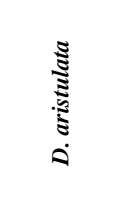 & 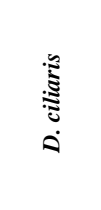 & 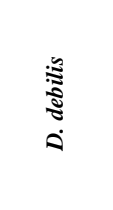 & 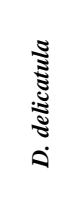 & 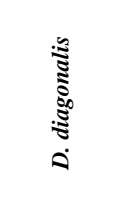 & $\begin{array}{l}\stackrel{\tilde{Z}}{0} \\
\dot{0} \\
\dot{0}\end{array}$ & 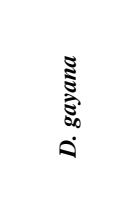 & 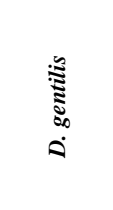 & 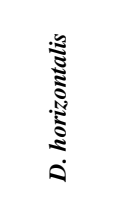 & 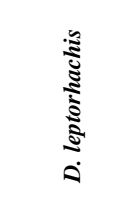 & 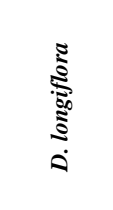 & 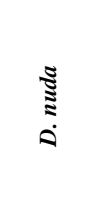 & 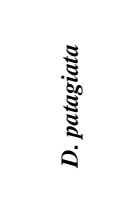 & 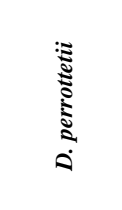 & $\frac{\Xi}{5}$ & 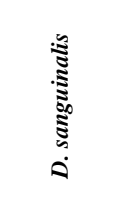 & 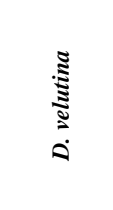 \\
\hline Type & Caryopse & Caryopse & Caryopse & $\begin{array}{c}\text { Caryops } \\
\mathrm{e}\end{array}$ & Caryopse & $\begin{array}{l}\text { Caryo } \\
\text { pse }\end{array}$ & Caryopse & Caryopse & Caryopse & Caryopse & Caryopse & Caryopse & Caryopse & $\begin{array}{c}\text { Caryo } \\
\text { pse }\end{array}$ & Caryopse & Caryopse & $\begin{array}{c}\text { Caryops } \\
\mathrm{e}\end{array}$ & Caryopse & $\begin{array}{c}\text { Caryops } \\
\mathrm{e}\end{array}$ \\
\hline Forme & $\begin{array}{l}\text { linéaire- } \\
\text { oblongue }\end{array}$ & $\begin{array}{l}\text { oblongue- } \\
\text { lancéolée }\end{array}$ & oblongue & $\begin{array}{c}\text { oblongu } \\
\mathrm{e}\end{array}$ & $\begin{array}{c}\text { ovée- } \\
\text { oblongue }\end{array}$ & $\begin{array}{l}\text { ellipti } \\
\text { que }\end{array}$ & elliptique & ovoïde & $\begin{array}{c}\text { ovée- } \\
\text { oblongue }\end{array}$ & elliptique & oblongue & elliptique & elliptique & $\begin{array}{l}\text { oblong } \\
\text { ue }\end{array}$ & $\begin{array}{l}\text { oblongue- } \\
\text { lancéolée }\end{array}$ & elliptique & $\underset{\mathrm{e}}{\text { elliptiqu }}$ & lancéolée & oblongue \\
\hline Sommet & aigu & aigu & aigu & aigu & aigu & aigu & aigu & aigu & rostré & aigu & $\begin{array}{c}\text { aigu- } \\
\text { acuminé }\end{array}$ & aigu & aigu & aigu & aigu & aigu & aigu & aigu & aigu \\
\hline $\begin{array}{l}\text { Longueur } \\
(\mathbf{m m})\end{array}$ & $2-3$ & $1,5-1,8$ & $\begin{array}{c}1,5 \\
\text { environ }\end{array}$ & $\begin{array}{c}2,5 \\
\text { environ }\end{array}$ & 1,2 & $1,5-2$ & $\begin{array}{c}1-1,5 \\
\text { environ }\end{array}$ & $\begin{array}{c}1,5 \\
\text { environ }\end{array}$ & 1,2 environ & $\begin{array}{c}1 \\
\text { environ }\end{array}$ & $\begin{array}{c}1,5-2 \\
\text { environ }\end{array}$ & 1,5 environ & 1 environ & $\begin{array}{c}1,5 \\
\text { enviro } \\
n\end{array}$ & $\begin{array}{c}1,5-2 \\
\text { environ }\end{array}$ & 1 environ & $1,5-2$ & $\begin{array}{c}1 \\
\text { environ }\end{array}$ & $\begin{array}{c}1,5 \\
\text { environ }\end{array}$ \\
\hline Couleur & blanchâtre & $\begin{array}{l}\text { brun foncé à } \\
\text { noir }\end{array}$ & blanchâtre & $\begin{array}{l}\text { brun } \\
\text { clair }\end{array}$ & $\begin{array}{c}\text { gris } \\
\text { pourpré }\end{array}$ & $\begin{array}{c}\text { noirât } \\
\text { re }\end{array}$ & $\begin{array}{l}\text { brun foncé } \\
\text { à noir }\end{array}$ & $\begin{array}{c}\text { blanc, } \\
\text { jaune ou } \\
\text { pourpre }\end{array}$ & $\begin{array}{l}\text { jaunâtre ou } \\
\text { brune }\end{array}$ & $\begin{array}{l}\text { beige } \\
\text { clair }\end{array}$ & $\begin{array}{l}\text { beige } \\
\text { clair }\end{array}$ & gris pourpré & $\begin{array}{c}\text { brun pâle } \\
\text { ou gris } \\
\text { pâle }\end{array}$ & $\begin{array}{c}\text { gris } \\
\text { clair à } \\
\text { brun }\end{array}$ & gris pourpré & brun pâle & $\begin{array}{c}\text { brun } \\
\text { foncé à } \\
\text { noir }\end{array}$ & brun clair & $\begin{array}{l}\text { brun } \\
\text { clair }\end{array}$ \\
\hline
\end{tabular}

Tableau 8 : Clé de détermination des espèces du genre Digitaria présentes au Sénégal.

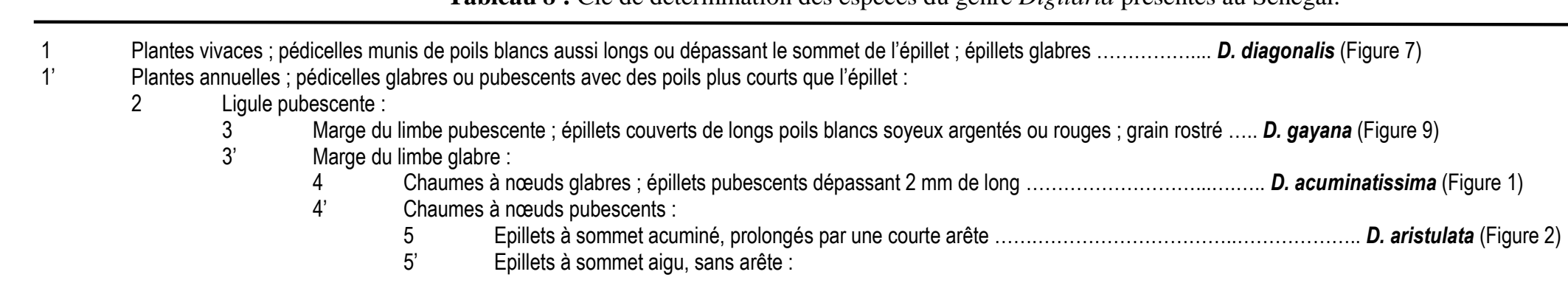


Marge du limbe pubescente ; glume supérieure 5-nervée

D. leptorhachis (Figure 12)

$\begin{array}{ll}7 & \text { Marge du limbe pubescent } \\ 7 & \end{array}$

$8 \quad$ Inflorescence composée d'un seul racème ; lemma inférieure avec 5 nervures

D. patagiata (Figure 15)

8' Inflorescence composée de 2 ou de plusieurs racèmes :

9 Inflorescence à 5 racèmes au plus :

$10 \quad$ Epillets glabres; chaumes à entre-nœuds glabres ; plante cultivée .................................. D. exilis (Figure 8$)$

$\begin{array}{ll}10 & \text { Epillets pubescents; chaum } \\ 9 & \text { Inflorescence à plus de } 5 \text { racèmes : }\end{array}$

11 Racèmes digités ou subdigités, disposés sur un axe commun ;

$12 \quad$ Epillets groupés par 2 (géminés):

13 Glume supérieure aussi longue que l'épillet :

$14 \quad$ Glume supérieure 3-nervée, lemma inférieure 5-nervée ........... D. gentilis (Figure 10)

14' Glume supérieure 5-7-nervée, lemma inférieure 7-nervée.............. D. debilis (Figure 5

$13^{\prime}$ Glume supérieure inférieure à l'épillet ;

15 Glume supérieure ne dépassant pas la moitié de l'épillet :

16 Lemma inférieure elliptique ; lemma supérieure à sommet

aigu .................................................. D. horizontalis (Figure 11)

16' Lemma inférieure ovale-oblongue ; lemma supérieure à sommet acuminé ...................................... D. sanguinalis (Figure 17)

15' Glume supérieure dépassant la moitié de l'épillet:

$17 \quad$ Glume inférieure ovale, grain long de $2 \mathrm{~mm}$........ D. ciliaris (Figure 4)

17' Glume inférieure absente ou en bourrelet, grain long de $1,5 \mathrm{~mm}$ :

18 Epillets se chevauchant sur les $1 / 4-1 / 2$ de leur longueur....

$18, \quad$ Epillets se chevauchant sur les $2 / 3$ de leur longueur velutina (Figure 19)

Epillets se chevauchant sur les $2 / 3$ de leur longueur

D. nuda (Figure 14)

12' Epillets groupés par 3 ou 4, à poils denses spatulés au sommet D. ternata (Figure 18)

11' Racèmes disposés en plusieurs verticilles (souvent plus de 6) sur un axe commun ; glume inférieure absente 


\section{DISCUSSION}

Il ressort de cette étude que le genre Digitaria est très complexe du fait de nombreux caractères qui varient souvent au sein de l'espèce. Ces caractères créent des formes de transition continues qui rendent difficile l'identification des espèces. C'est la raison pour laquelle les caractères les plus utilisés pour la distinction des espèces sont ceux de l'appareil reproducteur, plus particulièrement de l'épillet. Les caractéristiques de la pubescence de l'épillet, base de la classification infragénérique du genre Digitaria (Henrard, 1950), sont d'une grande importance taxonomique permettant de faciliter considérablement l'identification des espèces comme l'ont précisé Gilani et al. (2003). Lorsqu'ils existent, les poils de l'épillet sont uniquement situés au niveau de sa glume supérieure et de sa lemma inférieure. Ils sont absents sur la glume inférieure et la lemma supérieure, confirmant ainsi les résultats de Giraldo-Cañas (2004). Aussi, apparaît-il que les longueurs relatives de la glume supérieure et de la lemma inférieure sont très intéressantes et peuvent être étudiées pour distinguer des espèces très semblables.

Malgré toutes ces spécificités, l'identification des espèces sur la base de la seule morphologie de l'épillet reste très complexe et délicate. D'où l'importance de chercher d'autres caractères discriminants sur l'appareil végétatif de la plante. Cette étude a ainsi montré que les caractères des organes de l'appareil végétatif, souvent négligés à tort du fait de leur flexibilité suivant les conditions du milieu, offrent des caractères discriminants qui, quand ils sont corrélés avec d'autres, peuvent contribuer à une meilleure identification des espèces du genre Digitaria. Bien qu'ils soient peu utilisés, des caractères végétatifs tels que le cycle biologique, la pubescence de la ligule, de la marge du limbe, des nœuds du chaume sont plus faciles à observer et permettent de créer des groupes très francs. De plus, la pubescence des entrenœuds du chaume peut être étudiée chez des espèces proches morphologiquement comme c'est le cas de $D$. exilis et $D$. longiflora.

A partir des résultats consignés dans les différents tableaux (1 à 7), une clé de détermination (Tableau 8) est proposée pour servir d'outil d'aide à une meilleure identification des 19 espèces de Digitaria présentes au Sénégal.

\section{Conclusion}

Cette étude qui avait pour objectif d'élaborer une clé de détermination pour une meilleure identification des espèces du genre Digitaria répertoriées au Sénégal a permis de reconnaître qu'à partir des caractères de l'appareil reproducteur comme de l'appareil végétatif, les différentes espèces étudiées peuvent être discriminées. Les caractères de l'épillet, base de la classification infragénérique, sont essentiels pour l'identification des espèces. Néanmoins, même s'ils sont peu utilisés du fait de leur flexibilité suivant les conditions du milieu, des caractères végétatifs tels que la pubescence de la ligule, de la marge du limbe, des nœuds et des entre-nœuds du chaume sont plus faciles à observer et permettent de reconnaître plus aisément les espèces sur le terrain. Une étude phylogénétique basée sur des données morphologiques et moléculaires pourrait permettre de mieux comprendre les relations interspécifiques jusqu'ici implicites.

\section{REMERCIEMENTS}

Ce travail a été réalisé grâce à l'appui matériel des Herbiers de DAKAR (Département de Biologie Végétale, Faculté des Sciences et Techniques de l'Université Cheikh Anta Diop de Dakar) et de l'IFAN (Institut Fondamental d'Afrique Noire). Nos remerciements s'adressent aussi au Professeur Diego GIRALDO-CAÑAS (Université Nationale de Colombie) et au programme PPAAO/WAAPP et au projet WAAPP-fonio pour leur contribution.

\section{REFERENCES}

Adoukonou-Sagbadja H, Dansi A, Vodouhè R, Akpagana K. 2006. Indigenous knowledge and traditional conservation of fonio millet (Digitaria exilis, Digitaria iburua) in Togo. Biodiversity and Conservation, 15: 2379-2395. DOI : 10.1007/s10531-1-004-2938-3

Bâ AT, Noba K. 2001. Flore et biodiversité végétale au Sénégal. Science et Changements Planétaires/Sécheresse, 12(3): 149-155.

Bassène C, Mbaye MS, Camara AA, KANE A, Guèye M, Sylla SN, Sambou B, Noba 
K. 2014. La flore des systèmes agropastoraux de la Basse Casamance (Sénégal) : cas de la communauté rurale de Mlomp. Int. J. Biol. Chem. Sci., 8(5): 2258-2273. DOI : http://dx.doi.org/ 10.4314/ijbcs.v8i5.28

Bassène C, Mbaye MS, Camara AA, KANE A, Diangar S, Noba K. 2012. Flore adventice du maïs (Zea mays L.) dans le sud du Bassin arachidier (Sénégal) : structure et nuisibilité des espèces. Int. $J$. Appl. Biosci., 59: 4307-4320.

Berhaut J. 1967. Flore du Sénégal (édn $2^{\text {ème }}$ ). Editions Clairafrique: Dakar, Sénégal.

Gilani SS, Khan MA, Shinwari ZK, Hussain F, Yousaf Z. 2003. Taxonomic relationship of the genus Digitaria in Pakistan. Pakistan Journal of Botany, 35(3): 261-278.

Giraldo-Cañas D. 2004. Características micromorfológicas y anatómicas de la espiguilla y el antecio superior del género Digitaria (Poaceae: Panicoideae: Paniceae). Caldasia, 26: 1-35.

Henrard JT. 1950. Monograph of the Genus Digitaria. Leiden University: Netherland.

Hooker NB. 2009. Grasses of James Cook University, Townsville Campus. (Part B) Generic Descriptions and Key to Species. James Cook University: Townsville.

Lebrun JP, Stork A. 1991. Enumération des Plantes à Fleurs d'Afrique Tropicale (vol I, II, III, IV). Edition des conservatoires et jardin botaniques de la ville de Genève : Genève.

Mbaye MS. 2013. Association mil [Pennisetum glaucum (L.) R.Br] et niébé [Vigna unguiculata (L.) Walp.]: arrangement spatiotemporel des cultures, structure, dynamique et concurrence de la flore adventice et proposition d'un itinéraire technique. Thèse de Doctorat d'Etat, Université Cheikh Anta Diop, Dakar, p. 236.

Merchán MM. 2006. Acerca de la identifidad de Digitaria andicola y Digitaria cuatrecasasii (Poaceae: Panicoideae: Paniceae). Caldasia, 28(1): 9-13.

Noba K. 2002. La flore adventice dans le sud du basin arachidier (Sénégal) : Structure, dynamique et impact sur la production du mil et de l'arachide. Thèse de Doctorat d'Etat, Université Ckeikh Anta Diop, Dakar. p. 137.

Poilecot P. 1999. Les Poaceae du Niger (Vol. 56). Conservatoire et Jardin Botanique de la Ville de Genève, IUCN, CIRAD: BOI SSIERA.

Poilecot P. 1995. Les Poaceae de la Côte d'Ivoire (Vol. 50). Conservatoire et Jardin Botanique de la Ville de Genève, IUCN, CIRAD: BOISSIERA.

Quattrocchi U. 2006. CRC World Dictionary of Grasses: Common Names, Scientific Names, Eponyms, Synonyms, and Etymology. CRC Press: London.

USAID/Sénégal. 2008. Chaine de Valeur fonio - Sénégal : Analyse et Cadre Stratégique d'Initiatives pour la Croissance de la Filière. United States Agency for International Development.

Vanden Berghen C. 1991. Monocotylédones et Ptéridophytes. Dans Flore illustrée du Sénégal (Vol. X). Ministère du Développement rural et de l'Hydraulique, Direction des Eaux et Forêts, Dakar, Sénégal.

Vega AS, Rua GH, Fabbri LT, Rúgolo de Agrasar ZE. 2009. A morphology-based cladistic analysis of Digitaria (Poaceae, Panicoideae, Paniceae). Syst. Bot., 34(2): 312-323.

Vega A, Rúgolo de Agrasar Z. 2002b. Digitaria killeenii (Poaceae: Panicoideae: Paniceae), a new species from Bolivia. Syst. Bot., 27: 252-256.

Vodouhè SR, Zannou A, Achigan Dako E. 2003. Actes du premier atelier sur la diversité génétique du fonio (Digitaria exilis Stapf.) en Afrique de l'Ouest. Institut International des Ressources Phytogénétiques (IPGRI):Rome, Italie. 